\title{
ON A SHAPE CONTROL PROBLEM FOR THE STATIONARY NAVIER-STOKES EQUATIONS
}

\author{
Max D. Gunzburger ${ }^{1}$, Hongchul $\mathrm{KIm}^{2}$ and Sandro Manservisi ${ }^{3}$
}

\begin{abstract}
An optimal shape control problem for the stationary Navier-Stokes system is considered. An incompressible, viscous flow in a two-dimensional channel is studied to determine the shape of part of the boundary that minimizes the viscous drag. The adjoint method and the Lagrangian multiplier method are used to derive the optimality system for the shape gradient of the design functional.
\end{abstract}

Mathematics Subject Classification. 49K40, 76D05.

Received: April 12, 2000. Revised: September 23, 2000.

\section{INTRODUCTION}

Optimal shape control problems associated with the Navier-Stokes equations have a wide and important range of applications. Aerodynamic and hydrodynamic problems such as the design of cars, airplanes, and jet engines provide a few settings. Despite the fact that this field has been extensively studied, determining the best shape for a system governed by the Navier-Stokes equations is still a difficult and time consuming task.

Early studies devoted to optimal shape control problems for the Navier-Stokes equations can be found in $[28,29]$. Nowadays shape optimization problems are covered in several monographs (see, e.g., $[16,26,30,35])$ which, for the most part, focus on the case of the Laplace operator or Stokes flow. The theoretical treatment of optimal shape design problems are concerned with questions of existence, regularity of solutions, and differentiability properties. Also, the numerical implementation, the analysis, and the consistency of discrete approximations are fundamental issues. Some papers on the existence and regularity of solutions are $[6,9,20,28,32,33,36]$. However, those results generally lack a coherent first-order necessary condition and often the regularity assumed cannot be used in numerical algorithms. Other papers, e.g., $[4,12,13,17,25,27,33]$, deal with re-formulations of the problem, mainly to simplified situations. Again, results are far from complete.

In $[25,27,34]$ an interesting embedding domain technique is explored which provides an equivalent formulation of the optimization problem on a fixed domain. In particular, in [34], an explicit formula for shape variations for the Navier-Stokes equation is proposed. In this paper, we not only recover this result but extend it in the framework of a more general Lagrange multiplier technique that can easily take into account other constraints.

\footnotetext{
Keywords and phrases. Shape control, optimal design, Navier-Stokes equations, drag minimization.

1 Department of Mathematics, Iowa State University, Ames IA, 50011-2064, USA. e-mail: gunzburg@math.iastate.edu

Supported in part by the Air Force Office of Scientific Research under grant number F49620-95-1-0407.

2 Department of Mathematics, Kangnŭng National University, Kangnŭng 210-702, Korea.

e-mail: hongchul@knusun.kangnung.ac.kr

3 Department of Mathematics, Kaiserslautern University, Kaiserslautern, 67663, Germany. Current address: LIN, DIENCA, University of Bologna, Via dei colli 16, 40136 Bologna, Italy. e-mail: sandro.manservisi@mail.ing.unibo.it

Supported by the European community under grant XCT-97-0117.
} 
This paper is concerned with a shape optimization problem for the stationary incompressible Navier-Stokes equations and will cover some of the above topics. The main focus is to find an appropriate formulation of the optimal shape design problem that is attractive for consistent numerical computations. We consider the full Navier-Stokes case and recover the first-order necessary conditions that can be used for developing a consistent numerical approach to the problem. In contrast to previous authors, we make consistent use of the dual variables and introduce the regularity of the boundary explicitly as a main constraint which is enforced through Lagrange multiplier techniques. The resulting optimality condition is a system of equations and variational inequalities which express the compact and coherent mathematical formulation of the problem. Issues related to numerical approximations such as mesh adaptation, embedding domain techniques, and computational tests are fundamental, but due to space limitations, will be treated in future papers; the interested reader can also consult $[8,12,13,25-27,30]$ and the references cited therein.

Although we deal with a specific, two-dimensional drag minimization problem, the approach used here is discussed in general terms and can be used for many other optimal control problems involving different objective functionals and classes of shape controls. Furthermore, although the geometry is somewhat simple, our results can be extended to a general settings without further complications. Our aim here is to provide a systematic analysis for the problem in which the viscous drag is minimized through the use of variational methods and to derive a useful formula for the shape gradient of the design functional.

\subsection{The model shape control problem}

We consider the two-dimensional incompressible flow of a viscous fluid through the channel $\Omega$ shown in Figure 1. The velocity $\vec{u}$ and the pressure $p$ satisfy the stationary Navier-Stokes system

$$
\begin{array}{rlll}
-\nu \Delta \vec{u}+(\vec{u} \cdot \nabla) \vec{u}+\nabla p & =\vec{f} & \text { in } \Omega \\
\nabla \cdot \vec{u} & =0 & \text { in } \Omega
\end{array}
$$

along with the Dirichlet boundary conditions

$$
\vec{u}=\vec{g}= \begin{cases}\vec{g}_{1} & \text { on } \Gamma_{1} \\ \vec{g}_{2} & \text { on } \Gamma_{3} \\ \overrightarrow{0} & \text { on } \Gamma_{2} \cup \Gamma_{4}\end{cases}
$$

where $\vec{f}$ is the given body force. In (1.1), $\nu$ denotes the inverse of the Reynolds number whenever the variables are appropriately nondimensionalized. The vectors $\vec{g}_{1}$ and $\vec{g}_{2}$ are the given velocities at the inflow $\Gamma_{1}$ and outflow $\Gamma_{3}$ of the channel, respectively. Along the bottom, $\Gamma_{4}$, and the top, $\Gamma_{2}$, of the channel the velocity vanishes. The function $\vec{g}$ must satisfy the compatibility condition

$$
\int_{\Gamma} \vec{g} \cdot \vec{n} \mathrm{~d} s=0
$$

where $\vec{n}$ is the unit normal vector along the surface $\Gamma$.

If some other types of boundary conditions, e.g., natural boundary conditions or outflow boundary conditions, are specified along the left or right or bottom boundaries, the results given in this paper are formally valid but some technical details in the analysis should be carefully revised. The set $\Gamma_{\alpha}=\left\{\vec{x}=(x, z) \in \mathbb{R}^{2} \mid x \in(a, b), z=\right.$ $\alpha(x)\} \subset \Gamma_{2}$ is the shape which is to be determined through the optimization process. In the rest of the paper, we shall denote the interval $(a, b)$ by $I$ and the domain $\Omega$ by $\Omega(\alpha)$.

There is a substantial literature discussing the set of all possible shapes. Clearly, the function $\alpha$ must belong to $C^{0,1}(I)$ in order to be regular enough to suppress excessive oscillations; see, e.g., [29]. However, $\alpha \in C^{0,1}(I)$ may not be sufficient to enable one to explicitly derive a first-order necessary condition. Thus, here, the set of all admissible controls $\alpha$ is restricted to more regular shapes, namely, to belong to $C^{1,1}(I)$. In order to have a regular flow which is shape differentiable, we need a domain with piecewise $C^{1,1}$ boundary and convex corners 


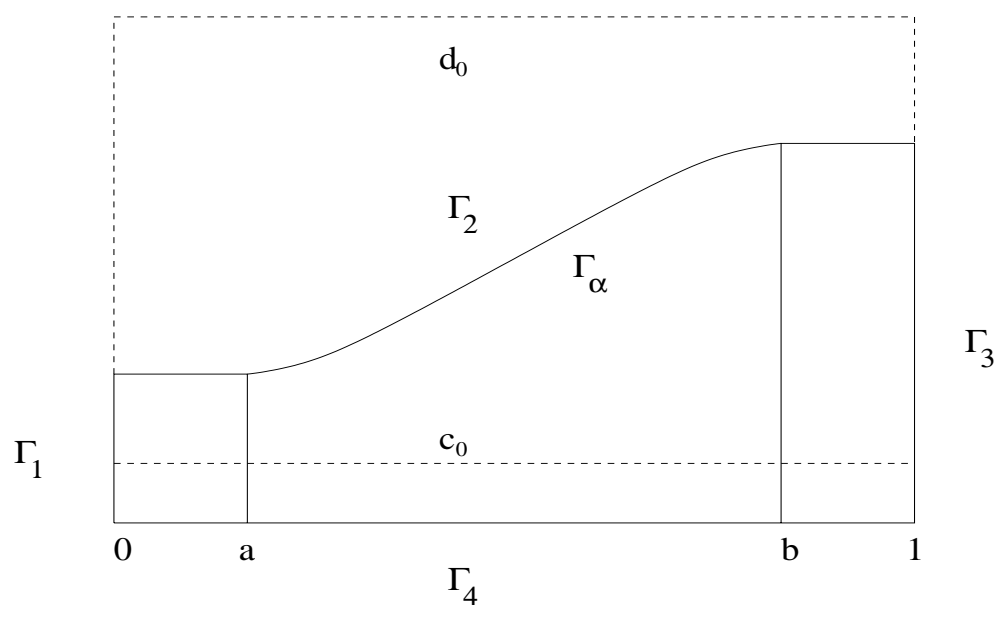

Figure 1. The flow domain $\Omega(\alpha)$ and its boundary; $\Gamma_{\alpha}$ denotes the part of the boundary whose shape is to be determined by the optimization process.

(for details see $[17,33]$ ). Furthermore, there are some practical constraints that may be taken into account; for example, the first derivative at the points $x=a$ and $x=b$ should be specified so that $\Gamma_{\alpha}$ is connected smoothly to the rest of the boundary.

Specifically, we define a set of allowable shapes in the following way. Let $c_{0}, d_{0}, c_{2}$, and $d_{2}$ be positive constants and $z_{1}$ and $z_{2}$ be the location of the controlled surface $\Gamma_{\alpha}$ at $x=a$ and $x=b$, respectively. Then, the set

$$
\left\{\alpha(x) \in C^{1}(I) \quad \mid \quad c_{i} \leq \alpha^{(i)} \leq d_{i} \text { for } i=0,2, \alpha(a)=z_{1}, \alpha(b)=z_{2}, \text { and } \alpha^{(1)}(a)=\alpha^{(1)}(b)=0\right\}
$$

may be a suitable set of allowable shapes, where $\alpha^{(i)}$ denotes the $i$ th derivative of $\alpha$. We have fixed the values of the first derivatives at $x=a$ and $x=b$ to be zero but they can be adjusted if the boundary segments $\Gamma_{2} \backslash \Gamma_{\alpha}$ are not horizontal when they meet $\Gamma_{\alpha}$.

In order to enforce the regularity of the boundary, we take $\alpha$ to be the solution of the Poisson equation

$$
\frac{\mathrm{d}^{2} \alpha}{\mathrm{d} x^{2}}=q \quad \text { on } \quad I, \quad \alpha(a)=z_{1}, \quad \text { and } \quad \alpha(b)=z_{2},
$$

where $c_{2} \leq q \leq d_{2}$ for all $x \in I$ is an unknown function. The other boundary conditions on $\alpha, i . e ., \alpha^{\prime}(a)=$ $\alpha^{\prime}(b)=0$, impose constraints on allowable functions $q$. In fact, if $\alpha$ and $q$ are related by $(1.5)$, then $\alpha^{\prime}(a)=$ $\alpha^{\prime}(b)=0$ if and only if

$$
\int_{a}^{b} q(x) \mathrm{d} x=0 \quad \text { and } \quad \int_{a}^{b} \int_{a}^{x} q(\xi) \mathrm{d} \xi \mathrm{d} x=z_{2}-z_{1}
$$

Since $q$ is bounded, we have that $\alpha \in C^{1}(I)$ and $\Gamma$ is piecewise $C^{1,1}$ with convex corners. We note that the lower bound for $\alpha$, i.e., $\alpha(x) \geq c_{0} \forall x \in I$, is necessary to avoid the intersection of $\Gamma_{2}$ and $\Gamma_{4}$ (see Fig. 1) and that the second derivative bounds are necessary to assure that the curvature on $\Gamma_{\alpha}$ can be computed.

One could examine several practical objective functionals for determining the shape of the boundary, e.g., the reduction of the drag due to viscosity or the identification of the velocity at a fixed vertical slit downstream. To fix ideas, we focus on the minimization of the cost functional, or, in the terminology of shape optimization, 
the design performance function,

$$
\mathcal{J}(\vec{u}, q, \alpha)=2 \nu \int_{\Omega(\alpha)} D(\vec{u}): D(\vec{u}) \mathrm{d} \vec{x}+\frac{\beta}{2} \int_{I} q^{2} \mathrm{~d} x
$$

where $\vec{u}$ is the velocity field defined on $\Omega(\alpha), D(\vec{u})=\frac{1}{2}\left(\nabla \vec{u}+(\nabla \vec{u})^{T}\right)$ is the deformation tensor for the flow $\vec{u}$, and $\beta$ is a nonnegative constant. For $\beta=0$, the functional (1.7) represents the rate of energy dissipation due to deformation and physically, except for an additive constant, can be identified with the viscous drag of the flow. We note that for a solenoidal flow field $\vec{u}$, we have $2 D(\vec{u}): D(\vec{u})=\nabla \vec{u}: \nabla \vec{u}$.

Formally speaking, the control problem we consider is to find $\vec{u}, \alpha$, and $q$ such that the functional (1.7) is minimized subject to the Navier-Stokes system (1.1)-(1.3) and the relations (1.5) and (1.6) being satisfied.

The plan of the rest of the paper is as follows. In the rest of this section, we introduce some notation and consider the associated boundary value problem for which the domain is given. In the next section, we give a precise description of the model optimization problem and then state and prove some results concerning the existence of optimal solutions. In Section 3, a first-order necessary condition is found through a direct sensitivity analysis and the adjoint equation method is used to compute the shape gradient for the model problem; the derivations of this section assume that the standard condition guaranteeing the uniqueness of solutions of the stationary Navier-Stokes system holds. In Section 4, we use the Lagrange multiplier method to obtain similar results; although the derivations of this section are not as direct, it does not require that the "uniqueness condition" holds.

\subsection{Notations}

In this paper, depending on the context, $C$ and $K$ denote generic constants whose values also depend on context. We denote by $H^{s}(\mathcal{O}), s \in \mathbb{R}$, the standard Sobolev space of order $s$ with respect to the set $\mathcal{O}$, which is either the flow domain $\Omega$, or its boundary $\Gamma$, or part of its boundary. Whenever $m$ is a nonnegative integer, the inner product over $H^{m}(\mathcal{O})$ is denoted by $(f, g)_{m}$ and $(f, g)$ denotes the inner product over $H^{0}(\mathcal{O})=L^{2}(\mathcal{O})$. Hence, we associate with $H^{m}(\mathcal{O})$ its natural norm $\|f\|_{m, \mathcal{O}}=\sqrt{(f, f)_{m}}$. For $1 \leq p<\infty$ the Sobolev space $W^{m, p}(\mathcal{O})$ is defined as the closure of $C^{\infty}(\mathcal{O})$ in the norm

$$
\|f\|_{W^{m, p}(\mathcal{O})}^{p}=\sum_{|\alpha| \leq m} \int_{\mathcal{O}}\left|\left(\frac{\partial}{\partial x}\right)^{\alpha} f(x)\right|^{p} \mathrm{~d} x .
$$

The closure of $C_{0}^{\infty}(\mathcal{O})$ under the norm $\|\cdot\|_{W^{m, p}(\mathcal{O})}$ will be denoted by $W_{0}^{m, p}(\mathcal{O})$. Whenever possible, we will neglect the domain label in the norm.

For vector-valued functions and spaces, we use boldface notation. For example, $\mathbf{H}^{s}(\Omega)=\left[H^{s}(\Omega)\right]^{n}$ denotes the space of $\mathbb{R}^{n}$-valued functions such that each component belongs to $H^{s}(\Omega)$. Of special interest is the space

$$
\mathbf{H}^{1}(\Omega)=\left\{v_{j} \in L^{2}(\Omega) \mid \frac{\partial v_{j}}{\partial x_{k}} \in L^{2}(\Omega) \quad \text { for } j, k=1,2\right\}
$$

equipped with the norm $\|\vec{v}\|_{1}=\left(\sum_{k=1}^{2}\left\|v_{k}\right\|_{1}^{2}\right)^{1 / 2}$. We define the space

$$
\mathbf{V}(\Omega)=\left\{\vec{u} \in \mathbf{H}^{1}(\Omega) \mid \nabla \cdot \vec{u}=0\right\}
$$

and the space of infinite differentiable solenoidal functions by

$$
\mathcal{V}(\Omega)=\left\{\vec{u} \in \mathbf{C}_{0}^{\infty}(\bar{\Omega}) \mid \nabla \cdot \vec{u}=0\right\} .
$$

For $\Gamma_{s} \subset \Gamma$ with nonzero measure, we also consider the subspace

$$
\mathbf{H}_{\Gamma_{s}}^{1}(\Omega)=\left\{\vec{v} \in \mathbf{H}^{1}(\Omega) \mid \vec{v}=\overrightarrow{0} \quad \text { on } \Gamma_{s}\right\} .
$$


Also, we write $\mathbf{H}_{0}^{1}(\Omega)=\mathbf{H}_{\Gamma}^{1}(\Omega)$. For any $\vec{v} \in \mathbf{H}^{1}(\Omega)$, we write $\|\nabla \vec{v}\|$ for the seminorm. Let $\left(\mathbf{H}_{\Gamma_{s}}^{1}\right)^{*}$ denote the dual space of $\mathbf{H}_{\Gamma_{s}}^{1}$. Note that $\left(\mathbf{H}_{\Gamma_{s}}^{1}\right)^{*}$ is a subspace of $\mathbf{H}^{-1}(\Omega)$, where the latter is the dual space of $\mathbf{H}_{0}^{1}(\Omega)$. The duality pairing between $\mathbf{H}^{-1}(\Omega)$ and $\mathbf{H}_{0}^{1}(\Omega)$ is denoted by $\langle\cdot, \cdot\rangle$.

Let $\vec{g}$ be an element of $\mathbf{H}^{1 / 2}(\Gamma)$. It is well known that $\mathbf{H}^{1 / 2}(\Gamma)$ is a Hilbert space with norm

$$
\|\vec{g}\|_{1 / 2, \Gamma}=\inf _{\vec{v} \in \mathbf{H}^{1}(\Omega) ; \gamma_{\Gamma} \vec{v}=\vec{g}}\|\vec{v}\|_{1}
$$

where $\gamma_{\Gamma}$ denotes the trace mapping $\gamma_{\Gamma}: \mathbf{H}^{1}(\Omega) \rightarrow \mathbf{H}^{1 / 2}(\Gamma)$. We let $\left(\mathbf{H}^{1 / 2}(\Gamma)\right)^{*}$ denote the dual space of $\mathbf{H}^{1 / 2}(\Gamma)$ and $\langle\cdot, \cdot\rangle_{\Gamma}$ denote the duality pairing between $\left(\mathbf{H}^{1 / 2}(\Gamma)\right)^{*}$ and $\mathbf{H}^{1 / 2}(\Gamma)$. From the definition of the dual norm, we have

$$
\|\vec{s}\|_{-1 / 2, \Gamma}=\sup _{\vec{g} \in \mathbf{H}^{1 / 2}(\Gamma) ; \vec{g} \neq \overrightarrow{0}} \frac{\langle\vec{s}, \vec{g}\rangle_{\Gamma}}{\|\vec{g}\|_{1 / 2}} .
$$

It is shown in [20] that

$$
\|\vec{s}\|_{-1 / 2, \Gamma}=\sup _{\vec{v} \in \mathbf{H}^{1}(\Omega) ; \vec{v} \neq \overrightarrow{0}} \frac{\left\langle\vec{s}, \gamma_{\Gamma} \vec{v}\right\rangle_{\Gamma}}{\|\vec{v}\|_{1}}
$$

provides an alternative and equivalent definition for the dual norm $\|\cdot\|_{-1 / 2}$.

Let $\Gamma_{s}$ be a smooth subset of $\Gamma$. Then, the trace mapping $\gamma_{\Gamma_{s}}: \mathbf{H}^{1}(\Omega) \rightarrow \mathbf{H}^{1 / 2}\left(\Gamma_{s}\right)$ is well defined and $\mathbf{H}^{1 / 2}\left(\Gamma_{s}\right)=\gamma_{\Gamma_{s}}\left(\mathbf{H}^{1}(\Omega)\right)$.

Since the pressure is only determined up to an additive constant by the Navier-Stokes system with velocity boundary conditions, we define the space of square integrable function having zero mean over $\Omega$ as

$$
L_{0}^{2}(\Omega)=\left\{p \in L^{2}(\Omega) \mid \int_{\Omega} p \mathrm{~d} \vec{x}=0\right\} .
$$

In order to define a weak form of the Navier-Stokes equations, we introduce the continuous bilinear forms

$$
a(\vec{u}, \vec{v})=2 \nu \sum_{i, j=1}^{2} \int_{\Omega} D(\vec{u}): D(\vec{v}) \mathrm{d} \vec{x} \quad \forall \vec{u}, \vec{v} \in \mathbf{H}^{1}(\Omega)
$$

and

$$
b(\vec{v}, q)=-\int_{\Omega} q \nabla \cdot \vec{v} \mathrm{~d} \vec{x} \quad \forall q \in L_{0}^{2}(\Omega), \quad \forall \vec{v} \in \mathbf{H}^{1}(\Omega)
$$

and the trilinear form

$$
c(\vec{w} ; \vec{u}, \vec{v})=\int_{\Omega} \vec{w} \cdot \nabla \vec{u} \cdot \vec{v} \mathrm{~d} \vec{x}=\sum_{i, j=1}^{2} \int_{\Omega} w_{j}\left(\frac{\partial u_{i}}{\partial x_{j}}\right) v_{i} \mathrm{~d} \vec{x} \quad \forall \vec{w}, \vec{u}, \vec{v} \in \mathbf{H}^{1}(\Omega) .
$$

Obviously, $a(\cdot, \cdot)$ is a continuous bilinear form on $\mathbf{H}^{1}(\Omega) \times \mathbf{H}^{1}(\Omega)$ and $b(\cdot, \cdot)$ is a continuous bilinear form on $\mathbf{H}^{1}(\Omega) \times L_{0}^{2}(\Omega)$; also $c(\cdot ; \cdot, \cdot)$ is a continuous trilinear form on $\mathbf{H}^{1}(\Omega) \times \mathbf{H}^{1}(\Omega) \times \mathbf{H}^{1}(\Omega)$ which can be verified by the Sobolev embedding of $\mathbf{H}^{1}(\Omega) \subset \mathbf{L}^{4}(\Omega)$ and Holder's inequality. We also have the coercivity property

$$
a(\vec{v}, \vec{v}) \geq C\|\vec{v}\|_{1}^{2} \quad \forall \vec{v} \in \mathbf{H}_{\Gamma_{s}}^{1}(\Omega)
$$

whenever $\Gamma_{s} \subset \Gamma$ has positive measure and the inf-sup condition

$$
\inf _{p \in L_{0}^{2}(\Omega)} \sup _{\vec{v} \in \mathbf{H}_{0}^{1}} \frac{b(\vec{v}, p)}{\|\vec{v}\|_{1}\|p\|} \geq K .
$$


For details concerning the function spaces we have introduced, one may consult $[2,5,37,38]$ and for details about the bilinear and trilinear forms and their properties, one may consult $[10,15,37]$.

Before continuing, we recall some notations and results about domains that will be of use in the sequel. We say that a domain $\Omega$ has a cusp at $x \in \Gamma$ if no affine image in $\bar{\Omega}$ of a finite cone has a vertex at $x$. If $\Omega$ is a Lipschitz continuous domain, the possibility of there being a cusp is excluded and therefore the domain $\Omega$ has the uniform extension property as the uniform Lipschitz sets are the open sets satisfying the cone property; see [9]. We recall the following extension theorem (Calderon's extension theorem); see [2].

Theorem 1.1. For every uniform Lipschitz domain $\Omega \subset \mathbb{R}^{2}$ and positive integer $m$, there exists a linear continuous extension operator

$$
E: \mathbf{H}^{m}(\Omega) \rightarrow \mathbf{H}^{m}\left(\mathbb{R}^{2}\right)
$$

such that for every $\vec{u} \in \mathbf{H}^{m}(\Omega)$ we have $\|E \vec{u}\|_{m} \leq C\|\vec{u}\|_{m}$, where the positive constant $C$ depends only on the cone imbedded in $\Omega$.

We recall also that a solenoidal extension to $\mathbb{R}^{2}$ of a solenoidal function defined in $\Omega$ can be found as described in [7]. In the rest of the paper, whenever it is not confusing, we denote the function and its extension by the same symbol. Since the function $\alpha$ defines $\Gamma_{\alpha}$, we write the corresponding domain and boundary as $\Omega(\alpha)$ and $\Gamma(\alpha)$ respectively. A useful result which shall be used in the existence theorem is as follows; see [14].

Theorem 1.2. Let $\Omega\left(\alpha_{m}\right)$ be a sequence having the cone property and that converges to $\Omega(\alpha)$. Let $\vec{u}_{m} \in$ $\mathbf{H}^{1}\left(\Omega\left(\alpha_{m}\right)\right)$ be a sequence converging to $\vec{u} \in \mathbf{H}^{1}(\Omega(\alpha))$. Assume that $\phi(s)$ is continuous, nonnegative, and convex for $s \in \mathbb{R}$. Then, we have that

$$
\int_{\Omega(\alpha)} \phi(\nabla \vec{u}) \mathrm{d} \vec{x} \leq \liminf _{m \rightarrow \infty} \int_{\Omega\left(\alpha_{m}\right)} \phi\left(\nabla \vec{u}_{m}\right) \mathrm{d} \vec{x} .
$$

\subsection{The associated boundary value problem}

We consider the formulation of the direct problem for the Navier-Stokes system (1.1)-(1.3) for which the boundary and all the data functions are known. Let $\Gamma(\alpha)$ be the boundary which includes the segment $\Gamma_{\alpha}$ (see Fig. 1) defined for a given $\alpha \in H^{2}(I)$. Given $\alpha$, we can compute $q$ by using (1.5).

A weak formulation of the Navier-Stokes system is given as follows:

$$
\begin{aligned}
& \text { given } \vec{f} \in \mathbf{H}^{-1}(\Omega(\alpha)) \text { and } \vec{g} \in \mathbf{H}^{1 / 2}(\Gamma(\alpha)) \text {, find }(\vec{u}, p) \in \mathbf{H}^{1}(\Omega(\alpha)) \times L_{0}^{2}(\Omega(\alpha)) \text { satisfying } \\
& \qquad\left\{\begin{array}{l}
a(\vec{u}, \vec{v})+c(\vec{u} ; \vec{u}, \vec{v})+b(\vec{v}, p)=\langle\vec{f}, \vec{v}\rangle \quad \forall \vec{v} \in \mathbf{H}_{0}^{1}(\Omega(\alpha)) \\
b(\vec{u}, q)=0 \quad \forall q \in L_{0}^{2}(\Omega(\alpha)) \\
\langle\vec{u}, \vec{s}\rangle_{\Gamma(\alpha)}=\langle\vec{g}, \vec{s}\rangle_{\Gamma(\alpha)} \quad \forall \vec{s} \in \mathbf{H}^{-1 / 2}(\Gamma(\alpha)) .
\end{array}\right.
\end{aligned}
$$

Existence and uniqueness results for solutions of the system (1.12) are contained in the following theorem; see, e.g., [37].

Theorem 1.3. Let $\Omega(\alpha)$ be an open, bounded set of $\mathbb{R}^{2}$ with Lipschitz-continuous boundary $\Gamma(\alpha)$. Let $\vec{f} \in$ $\mathbf{H}^{-1}(\Omega(\alpha))$ and $\vec{g} \in \mathbf{H}^{1 / 2}(\Gamma(\alpha))$ and let $\vec{g}$ satisfy the compatibility condition (1.4). Then,

(i) there exists at least one solution $(\vec{u}, p) \in \mathbf{H}^{1}(\Omega(\alpha)) \times L^{2}(\Omega(\alpha))$ of $(1.12)$;

(ii) the set of velocity fields that are solutions of $(1.12)$ is closed in $\mathbf{H}^{1}(\Omega(\alpha))$ and is compact in $\mathbf{L}^{2}(\Omega(\alpha))$; and

(iii) if

$$
\nu>\nu_{0}(\Omega(\alpha), \vec{f}, \vec{g})
$$


for some positive $\nu_{0}$ whose value is determined by the given data, then the set of solutions of (1.12) consists of a single element.

Note that solutions of (1.12) exists for any value of the Reynolds number. However, (iii) implies that uniqueness can be guaranteed only for "large enough" values of $\nu$ or for "small enough" data $\vec{f}$ and $\vec{g}$.

In order to write the first-order necessary condition that optimal solutions must satisfy, we shall need additional regularity for solutions of (1.12). To achieve the needed smoothness, we have to assume that the data of the problem is smoother than that assumed in the hypotheses of Theorem 1.3. In that case, we have the following results; see $[15,33,37,38]$.

Theorem 1.4. Let the hypotheses of Theorem 1.3 hold. Let $\Gamma(\alpha)$ be piecewise $C^{1,1}$ with convex corners, $\vec{g} \in$ $\mathbf{H}^{3 / 2}(\Gamma(\alpha))$, and $\vec{f} \in \mathbf{L}^{2}(\Omega(\alpha))$. Let $(\vec{u}, p)$ denote a solution of (1.12). Then,

(i) $(\vec{u}, p) \in \mathbf{H}^{2}(\Omega(\alpha)) \times H^{1}(\Omega(\alpha)) \cap L_{0}^{2}(\Omega(\alpha))$ and

(ii) the set of solutions of (1.12) is closed in $\mathbf{H}^{2}(\Omega(\alpha))$ and compact in $\mathbf{H}^{1}(\Omega(\alpha))$.

\section{The MOdel Shape CONTROL PROBlem AND EXISTENCE OF SOLUTIONS}

In this section we give a precise formulation of the shape control problem we consider and prove the existence of optimal solutions.

\subsection{Formulation of the optimal shape control problem}

We now formulate the model of the optimal shape control problem. We define the closed convex set

$$
\mathcal{Q}_{a d}=\left\{\alpha \in H^{2}(I) \mid 0<c_{0} \leq \alpha \leq d_{0} \quad \alpha(a)=z_{1}, \alpha(b)=z_{2}, \text { and } \alpha^{\prime}(a)=\alpha^{\prime}(b)=0\right\}
$$

and introduce the variable $q$ belonging to the set

$$
\mathcal{B}_{a d}=\left\{q \in L^{2}(I) \mid c_{2} \leq q \leq d_{2} \text { almost everywhere }\right\}
$$

defined by

$$
\int_{I} q v \mathrm{~d} x=\int_{I} \frac{\mathrm{d}^{2} \alpha}{\mathrm{d} x^{2}} v \mathrm{~d} x \quad \forall v \in L^{2}(I) .
$$

The constants $c_{2}$ and $d_{2}$ are such that the set $\mathcal{Q}_{a d}$ is not empty. From the Sobolev imbedding theorem, we have that $H^{2}(I) \subset C^{1}(\bar{I}) \subset C^{0,1}(\bar{I})$ and therefore, if $\alpha \in \mathcal{Q}_{a d}$ and $q \in \mathcal{B}_{a d}$, then $\alpha \in C^{1,1}$ at least.

The optimal shape control problem can then be stated in the following way:

Given $\vec{f} \in \mathbf{L}^{2}(\Omega(\alpha))$ and $\vec{g} \in \mathbf{H}^{3 / 2}(\Gamma(\alpha))$ satisfying the compatibility condition (1.4), find $(\widehat{u}, \widehat{p}, \widehat{q}, \widehat{\alpha})$ such that

$$
\mathcal{J}(\widehat{u}, \widehat{q}, \widehat{\alpha}) \leq \mathcal{J}(\vec{u}, q, \alpha)
$$

$$
\text { for all }(\vec{u}, p, q, \alpha) \in \mathbf{H}^{2}(\Omega(\alpha)) \times \mathbf{H}^{1}(\Omega(\alpha)) \cap L_{0}^{2}(\Omega(\alpha)) \times \mathcal{B}_{a d} \times \mathcal{Q}_{a d} \text { satisfying (1.12) and (2.1). }
$$

The extended domain $\widehat{\Omega}$ is defined to be the rectangle $[0,1] \times\left[0, d_{0}\right]$. We also assume that $\vec{f}$ is defined over the extended domain $\widehat{\Omega}$ and write $\vec{f}(\Omega(\alpha))$ to denote its restriction to $\Omega(\alpha)$.

The admissible set of states and controls is given by

$$
\begin{gathered}
\mathcal{A}_{a d}=\left\{(\vec{u}, p, q, \alpha) \in \mathbf{H}^{2}(\Omega(\alpha)) \cap \mathbf{V}(\Omega(\alpha)) \times H^{1}(\Omega(\alpha)) \cap L_{0}^{2}(\Omega(\alpha)) \times \mathcal{B}_{a d} \times \mathcal{Q}_{a d}\right. \\
\text { such that } \mathcal{J}(\vec{u}, q, \alpha)<\infty \text { and }(\vec{u}, p, q, \alpha) \text { satisfies }(1.12) \text { and }(2.1)\} .
\end{gathered}
$$




\subsection{Existence of optimal solutions}

We now turn to the question of the existence of optimal solutions for the problem in (2.2).

Theorem 2.1. There exists at least one optimal solution $(\vec{u}, p, q, \alpha) \in \mathcal{A}_{a d}$ of the optimal shape control problem (2.2).

Proof. The proof follows from standard techniques (see, e.g., [6] or [20]) and here we sketch the main idea. First, we prove that there exists a solution $(\vec{u}, p, q, \alpha)$ of the optimal control problem in $\mathbf{H}^{1}(\Omega(\alpha)) \times L_{0}^{2}(\Omega(\alpha)) \times \mathcal{B}_{a d} \times \mathcal{Q}_{a d}$ and then from the fact that the boundary is piecewise $C^{1,1}$ with convex corners, we conclude that this solution is in $\mathbf{H}^{2}(\Omega(\alpha)) \cap \mathbf{V}(\Omega(\alpha)) \times H^{1}(\Omega(\alpha)) \cap L_{0}^{2}(\Omega(\alpha)) \times \mathcal{B}_{a d} \times \mathcal{Q}_{a d}$.

Let $\widehat{\Omega}$ be the extended domain (in our case for example $\widehat{\Omega}=\left\{(x, z) \in \mathbb{R}^{2}: 0 \leq x \leq 1,0 \leq z \leq d_{0}\right\}$ ). On $\widehat{\Omega}-\Omega(\alpha)$, we can extend the velocity and the pressure fields according to Theorem 1.1 and denote them by $\widehat{u}$ and $\widehat{p}$, respectively. Also, on $\widehat{\Omega}-\Omega(\alpha)$ we define the extended body force $\widehat{f}$ by substituting the extended fields into the Navier-Stokes equations. We fix the test functions $\widehat{v}$ in $H_{0}^{1}(\widehat{\Omega})$ so that they are independent of boundary variations.

Consider the following procedure to construct the extension $(\widehat{u}, \widehat{p}, \widehat{f})$ of $(\vec{u}, p, \vec{f})$. Given $\Omega(\alpha)$ and $(\vec{u}, p, \vec{f})$ we set $\widehat{u}=\vec{u}$ and $\widehat{p}=p$ over $\Omega(\alpha)$ and zero over $\widehat{\Omega}-\Omega(\alpha)$. By using $(\widehat{u}, \widehat{p})$ and the Navier-Stokes operator we compute $\widehat{f}$ over the extended domain. Now $(\widehat{u}, \widehat{p}, \widehat{f})$ satisfies the Navier Stokes system over a fixed domain.

With this setting, the $(\widehat{u}, \widehat{p})$ satisfies $(1.12)$ on $\widehat{\Omega}$ for all admissible $\alpha$. The domain $\Omega(\alpha)$ lies inside the extended domain and therefore if the test functions $\widehat{v} \in \mathbf{H}_{0}^{1}(\widehat{\Omega}) \cap \mathbf{H}^{1}(\Omega(\alpha))$ are used, then, over $\Omega(\alpha)$, $(\widehat{u}, \widehat{p})$ satisfies

$$
\left\{\begin{array}{l}
a(\widehat{u}, \widehat{v})+c(\widehat{u} ; \widehat{u}, \widehat{v})+b(\widehat{v}, p)-\langle\vec{t}, \widehat{v}\rangle_{\Gamma_{2}}=\langle\widehat{f}, \widehat{v}\rangle \quad \forall \widehat{v} \in \mathbf{H}_{0}^{1}(\widehat{\Omega}) \cap \mathbf{H}^{1}(\Omega(\alpha)) \\
b(\widehat{u}, \widehat{q})=0 \quad \forall \widehat{q} \in L_{0}^{2}(\Omega(\alpha)) \cap L_{0}^{2}(\widehat{\Omega}) \\
\langle\widehat{u}, \widehat{s}\rangle_{\Gamma(\alpha)}=\langle\widehat{g}, \widehat{s}\rangle_{\Gamma(\alpha)} \quad \forall \widehat{s} \in \mathbf{H}^{-1 / 2}(\Gamma(\alpha)),
\end{array}\right.
$$

where $\vec{t}$ is defined by

$$
\vec{t}=-\widehat{p} \vec{n}+2 \nu D(\widehat{u}) \cdot \vec{n} \quad \text { on } \Gamma_{2}
$$

with $\vec{n}$ the unit vector normal to the curve $\Gamma_{2}$. It is clear that when $\widehat{f}$ is known over $\widehat{\Omega}$ then $\vec{t}$ is known and vice versa.

Since the set of admissible solutions $\mathcal{A}_{a d}$ is not empty and the set of the values assumed by the functional is bounded from below, there exists a minimizing sequence $\left(q_{m}, \alpha_{m}\right)$ in $\mathcal{B}_{a d} \times \mathcal{Q}_{a d}$ and a corresponding sequence of solutions over the extended domain $\left(\widehat{u}_{m}, \widehat{p}_{m}, \vec{t}_{m}\right) \in \mathbf{H}^{1}(\widehat{\Omega}) \times L_{0}^{2}(\widehat{\Omega}) \times \mathbf{H}^{-1 / 2}\left(\Gamma_{2}\right)$ which are uniformly bounded. Using a standard argument, we can extract subsequences $\left(\widehat{u}_{n}, \widehat{p}_{n}, \vec{t}_{n}, q_{n}, \alpha_{n}\right)$ such that, for some $(\widehat{u}, \widehat{p}, \vec{t}, q, \alpha) \in$ $\mathbf{H}^{1}(\widehat{\Omega}) \times L_{0}^{2}(\widehat{\Omega}) \times \mathbf{H}^{-1 / 2}\left(\Gamma_{2}\right) \times L^{2}(I) \times H^{2}(I)$, we have

$$
\begin{array}{lllll}
\widehat{u}_{n} & \rightarrow \widehat{u} & \text { weakly } & \text { in } \mathbf{H}^{1}(\widehat{\Omega}) \\
\widehat{u}_{n} & \rightarrow \widehat{u} & \text { strongly } & \text { in } \mathbf{L}^{2}(\widehat{\Omega}) \\
\gamma_{\Gamma(\alpha)} \widehat{u}_{n} & \rightarrow \gamma_{\Gamma(\alpha)} \widehat{u} & \text { weakly } & \text { in } \mathbf{H}^{1 / 2}(\Gamma(\alpha)) \\
\gamma_{\Gamma(\alpha)} \widehat{u}_{n} & \rightarrow \gamma_{\Gamma(\alpha)} \widehat{u} & \text { strongly } & \text { in } & \mathbf{L}^{2}(\Gamma(\alpha)) \\
\widehat{p}_{n} & \rightarrow \widehat{p} & \text { weakly } & \text { in } & L^{2}(\widehat{\Omega}) \\
\vec{t}_{n} & \rightarrow & \vec{t} & \text { weakly } & \text { in } \mathbf{H}^{-1 / 2}\left(\Gamma_{2}\right) \\
\alpha_{n} & \rightarrow \alpha & \text { weakly } & \text { in } & H^{2}(I) \\
q_{n} & \rightarrow q & \text { weakly } & \text { in } & L^{2}(I),
\end{array}
$$


where the strong convergence results follow from the Sobolev imbedding theorem. The limit $(\widehat{u}, \widehat{p}, \vec{t}, q, \alpha)$ belongs to $\mathbf{H}^{1}(\widehat{\Omega}) \times L_{0}^{2}(\widehat{\Omega}) \times \mathbf{H}^{-1 / 2}\left(\Gamma_{2}\right) \times \mathcal{B}_{a d} \times \mathcal{Q}_{a d}$ since the sets $\mathcal{B}_{a d}$ and $\mathcal{Q}_{a d}$ are convex and closed. We recall that every closed convex set is weakly closed (see [31]).

Now we wish to show that the restriction of $(\widehat{u}, \widehat{p}, \vec{t}, q, \alpha) \in \mathbf{H}^{1}(\widehat{\Omega}) \times L_{0}^{2}(\widehat{\Omega}) \times \mathbf{H}^{-1 / 2}\left(\Gamma_{2}\right) \times \mathcal{B}_{a d} \times \mathcal{Q}_{a d}$ to $\Omega(\alpha)$ is a solution of (2.2). It is enough to prove this for all the test functions $\widehat{v} \in \mathcal{V}(\widehat{\Omega})$ and then we claim the result for all $\widehat{v} \in \mathbf{H}_{0}^{1}(\widehat{\Omega})$ by a continuity argument. It is possible to show that the limit satisfies (2.3) simply by extending the integral to $\widehat{\Omega}$ and by applying standard Navier-Stokes results for fixed boundaries [1, 22,37]. For example for the first term (recalling that $\widehat{u}_{n}$ vanishes on $\widehat{\Omega}-\Omega\left(\alpha_{n}\right)$ and applying the above convergence results) we have

$$
\lim _{n \rightarrow \infty} \int_{\Omega\left(\alpha_{n}\right)} D\left(\vec{u}_{n}\right): D(\widehat{v}) \mathrm{d} \vec{x}=\lim _{n \rightarrow \infty} \int_{\widehat{\Omega}} D\left(\widehat{u}_{n}\right): D(\widehat{v}) \mathrm{d} \vec{x}=\int_{\widehat{\Omega}} D(\widehat{u}): D(\widehat{v}) \mathrm{d} \vec{x}=\int_{\Omega(\alpha)} D(\vec{u}): D(\widehat{v}) \mathrm{d} \vec{x},
$$

for all $\widehat{v} \in \mathcal{V}(\widehat{\Omega})$. The nonlinear term does not present problems since, once we are in the extended domain, the limit is assured by standard arguments on fixed domains $[1,37]$ if $\vec{u}_{n}$ converges weakly in $\mathbf{H}^{1}(\widehat{\Omega})$ and strongly in $L^{2}(\widehat{\Omega})$.

The formulation in (1.12) and in (2.3) are equivalent when the test functions are taken in $\mathbf{H}_{0}^{1}(\Omega(\alpha))$ and therefore the restriction $(\vec{u}, p, q, \alpha)$ in $\Omega(\alpha)$ of the limit $(\widehat{u}, \widehat{p}, q, \alpha)$ satisfies the Navier-Stokes equations in (1.12). Using Theorem 1.2, we have that the functional is weakly lower semi-continuous and consequently we have that $(\vec{u}, p, q, \alpha)$ is a solution of the optimal shape control problem in $\mathbf{H}^{1}(\Omega(\alpha)) \times L_{0}^{2}(\Omega(\alpha)) \times \mathcal{B}_{a d} \times \mathcal{Q}_{a d}$.

The regularity required by the theorem follows from simple considerations. From the fact that $\vec{g} \in \mathbf{H}^{1}(\Gamma(\alpha))$, it follows that $\vec{u}$ is in $\mathbf{H}^{3 / 2}(\Omega(\alpha))$ and the nonlinear term is in $L^{2}(\Omega(\alpha))$ at least. Since the boundary is piecewise $C^{1,1}$ with convex corners, we can apply the regularity results for the Stokes flow in [17,33] and conclude that this solution is in $\mathbf{H}^{2}(\Omega(\alpha)) \cap \mathbf{V}(\Omega(\alpha)) \times H^{1}(\Omega(\alpha)) \cap L_{0}^{2}(\Omega(\alpha)) \times \mathcal{B}_{a d} \times \mathcal{Q}_{a d}$.

\section{THE FIRST ORDER NECESSARY CONDITION}

\subsection{Preliminaries}

Let $\mathcal{C}_{a d}$ be the set of $\alpha \in \mathcal{Q}_{a d}$ such that $\alpha^{\prime \prime} \in \mathcal{B}_{a d}$. Let $\alpha \in \mathcal{C}_{a d}$ be the function that determines $\Gamma_{\alpha} \subset \Gamma(\alpha)$. After deformation, the domain $\Omega(\alpha)$ takes a new shape $\Omega(\widetilde{\alpha})$ with boundary $\Gamma(\widetilde{\alpha})$ corresponding to the function $\widetilde{\alpha} \in \mathcal{C}_{a d}$. The field, defined on $\Gamma_{\alpha}$, transforming $\Gamma_{\alpha}$ into $\Gamma_{\widetilde{\alpha}}$ is indicated by $\vec{V}$ and the corresponding variation by $\delta \alpha=\widetilde{\alpha}-\alpha$. For example, in our specific case, if $\alpha, \widetilde{\alpha} \in \mathcal{Q}_{a d}$, then $\delta \alpha=\widetilde{\alpha}-\alpha$ and $\vec{V}=(0, \delta \alpha)$, where the vector $\vec{V}$ is defined on $\Gamma_{\alpha}$. We note that, as proved in [35], the vector $\vec{V}$ can always be extended from $\Gamma_{\alpha}$ to every point $(x, z) \in \Omega(\alpha)$ or every point in $\mathbb{R}^{2}$, i.e. in our case we can simply take $\vec{V}(x, z)=(0, z \delta \alpha(x) / \alpha(x)) \in \mathbb{R}^{2}$.

We can generate a family of boundary parameterized by $t$ as

$$
\Gamma_{\alpha+t \delta \alpha}=\left\{\vec{x}_{\alpha}+t \vec{V}\left(\vec{x}_{\alpha}\right) \mid \vec{x}_{\alpha} \in \Gamma_{\alpha}\right\}
$$

for all $t \in[0,1]$. Let $\mathcal{K}(\alpha)$ be a functional on the domain $\Omega(\alpha)$. We say that $\mathcal{K}(\alpha): \mathcal{C}_{a d} \rightarrow \mathbb{R}$ is Gateauxdifferentiable at $\alpha$ in the direction $\delta \alpha$ if there is a real number $\mathcal{K}^{\prime}=(D \mathcal{K} / D \alpha) \delta \alpha$ such that

$$
\lim _{t \rightarrow 0+} \frac{\left|\mathcal{K}\left(\Omega\left(\alpha_{t}\right)\right)-\mathcal{K}(\Omega(\alpha))-t \mathcal{K}^{\prime}\right|}{t}=0
$$

In a similar way, when $Y$ is a Banach Space, the map $\vec{u}(\alpha): \mathcal{C}_{a d} \rightarrow Y$ is said to be Gateaux-differentiable at $\alpha$ in the direction $\delta \alpha(\vec{V}=(0, \delta \alpha))$ if there exists a function $\vec{u}^{\prime} \in Y(\Omega(\alpha))$ such that

$$
\lim _{t \rightarrow 0+} \frac{\left\|\vec{u}_{t}\left(\Omega\left(\alpha_{t}\right)\right)-\vec{u}(\Omega(\alpha))-t \vec{u}^{\prime}\right\|_{Y(\Omega(\alpha))}}{t}=0
$$


Before proving differentiability, we need the following lemma [35].

Lemma 3.1. Given $\alpha, \widetilde{\alpha}$ in $\mathcal{C}_{a d}(\delta \alpha=\widetilde{\alpha}-\alpha), \widehat{y}_{1} \in W^{1,1}\left(\mathbb{R}^{2}\right)$ and $\widehat{y}_{2} \in W^{2,1}\left(\mathbb{R}^{2}\right)$. Let

$$
\mathcal{K}_{\Omega(\alpha)}=\int_{\Omega(\alpha)} \widehat{y}_{1}(\vec{x}) \mathrm{d} \vec{x} \quad \text { and } \quad \mathcal{K}_{\Gamma(\alpha)}=\int_{\Gamma(\alpha)} \widehat{y}_{2}(s) \mathrm{d} s .
$$

Then,

$$
\begin{aligned}
\frac{D \mathcal{K}_{\Omega}}{D \alpha} \cdot \delta \alpha & =\int_{\Omega(\alpha)} \nabla \cdot\left(\vec{V} \widehat{y}_{1}(\vec{x})\right) \mathrm{d} \vec{x}=\int_{\Gamma_{\alpha}} \widehat{y}_{1}(s)(\vec{V} \cdot \vec{n}) \mathrm{d} s \\
\frac{D \mathcal{K}_{\Gamma}}{D \alpha} \cdot \delta \alpha & =\int_{\Gamma_{\alpha}}\left(\frac{\partial \widehat{y}_{2}}{\partial n}+\kappa \widehat{y}_{2}(s)\right)(\vec{V} \cdot \vec{n}) \mathrm{d} s
\end{aligned}
$$

where $\vec{V}$ is the vector $(0, \delta \alpha)$ defined on $\Gamma_{\alpha}$ and $\kappa$ and $\vec{n}$ are the curvature and the unit vector normal to $\Gamma_{\alpha}$, respectively.

This lemma has been stated in the context of our particular optimal control situation but a more general framework can be found in [30] and [35]. We remark that, in order to apply the above lemma, the functions $\widehat{y}_{1}$ and $\widehat{y}_{2}$ must be defined on $\mathbb{R}^{2}$ or extended outside $\Omega(\alpha)$ and its gradient must be integrable. This allows the limit to be represented as a boundary integral over $\Gamma_{\alpha}$. The requirement $\widehat{y}_{1} \in W^{1,1}\left(\mathbb{R}^{2}\right)$ implies a certain regularity for the solution of the Navier-Stokes system. If such a regularity is not present, we may extend the previous lemma to a function $\widehat{y}_{1}$ in $L^{2}\left(\mathbb{R}^{2}\right)$. In this case, the functional is weakly differentiable in $H^{-2}$ [35], which may not imply regular solutions and its representation as a boundary integral on $\Gamma_{\alpha}$.

\subsection{Differentiability of candidate optimal solutions and the functional}

We shall show that the optimal control solution must satisfy a first-order necessary condition which leads to a variational inequality. By studying this variational inequality, a possible candidate for the optimal control solution can be found. In order to obtain this result, we need to prove the differentiability of the functional, and before that, of functions satisfying the constraints.

Theorem 3.2. Let (1.13) hold so that (1.12) has a unique solution. Let $(\vec{u}, p, q, \alpha) \in \mathcal{A}_{a d}$. The mapping $(\vec{u}, p)=(\vec{u}(\alpha), p(\alpha))$ has a Gateaux derivative $\left(\vec{u}^{\prime}, p^{\prime}\right)=\left(\frac{D \vec{u}}{D \alpha} \cdot \delta \alpha, \frac{D p}{D \alpha} \cdot \delta \alpha\right)$ in every direction $\delta \alpha=\widetilde{\alpha}-\alpha$ for all $\widetilde{\alpha}$ in $\mathcal{A}_{a d}$. Furthermore, $\left(\vec{u}^{\prime}, p^{\prime}\right)$ is the solution of the problem

$$
\left\{\begin{array}{l}
\nu a\left(\vec{u}^{\prime}, \vec{v}\right)+c\left(\vec{u} ; \vec{u}^{\prime}, \vec{v}\right)+c\left(\vec{u}^{\prime} ; \vec{u}, \vec{v}\right)+b\left(\vec{v}, p^{\prime}\right)=0 \quad \forall \vec{v} \in \mathbf{H}_{0}^{1}(\Omega(\alpha)) \\
b\left(\vec{u}^{\prime}, q\right)=0 \quad \forall q \in L_{0}^{2}(\Omega(\alpha)) \\
\int_{\Gamma(\alpha)} \vec{s} \cdot\left(\vec{u}^{\prime}+\frac{\partial \vec{u}}{\partial n}(\vec{V} \cdot \vec{n})\right) \mathrm{d} s=0 \quad \forall \vec{s} \in \mathbf{H}^{-1 / 2}(\Gamma(\alpha))
\end{array}\right.
$$

where the vector $\vec{V}$, defined by $(0, \delta \alpha)$ on $\Gamma_{\alpha}$, vanishes on $\Gamma(\alpha)-\Gamma_{\alpha}$. The unit vector $\vec{n}$ is the unit normal to the boundary $\Gamma(\alpha)$.

Proof. Let $\widehat{\Omega}$ be the extended domain (in our case $\widehat{\Omega}$ may be $\left\{(x, z) \in \mathbb{R}^{2}: 0 \leq x \leq 1,0 \leq z \leq d_{0}\right\}$ ). On $\widehat{\Omega}-\Omega(\alpha)$ we can extend the velocity and the pressure fields by the Calderon theorem and denote them by $\widehat{u}$ and $\widehat{p}$ respectively. We assume that the body force $\vec{f} \in \mathbf{L}^{2}(\widehat{\Omega})$ and the test functions $\widehat{v} \in \mathbf{H}_{0}^{1}(\widehat{\Omega})$ are defined over the extended domain so that they are independent of boundary variations.

Let $(\widehat{u}, \widehat{p}, q, \alpha)$ and $\left(\widehat{u}_{1}, \widehat{p}_{1}, q_{1}, \widetilde{\alpha}\right)$ be given in $\mathcal{A}_{a d}$. We set $\delta \alpha=\widetilde{\alpha}-\alpha$ and $\alpha_{t}=\alpha+t \delta \alpha$. The domain $\Omega(\alpha)$ lies inside the extended domain and therefore, if the test functions $\widehat{v} \in \mathbf{H}_{0}^{1}(\widehat{\Omega}) \cap \mathbf{H}^{1}(\Omega(\alpha))$ are used, then 
$(\widehat{u}, \widehat{p}, q, \alpha) \in \mathcal{A}_{a d}$ satisfies

$$
\left\{\begin{array}{l}
\int_{\Omega(\alpha)}\left(-\nu \nabla^{2} \widehat{u}+(\widehat{u} \cdot \nabla) \widehat{u}+\nabla \widehat{p}-\vec{f}\right) \cdot \widehat{v} \mathrm{~d} \vec{x}=0 \quad \forall \widehat{v} \in \mathbf{H}_{0}^{1}(\widehat{\Omega}) \cap \mathbf{V}(\Omega(\alpha)) \\
\int_{\Omega(\alpha)} \widehat{q} \nabla \cdot \widehat{u} \mathrm{~d} \vec{x}=0 \quad \forall \widehat{q} \in L_{0}^{2}(\widehat{\Omega}) \cap L_{0}^{2}(\Omega(\alpha)) \\
\int_{\Gamma(\alpha)}(\widehat{u}-\vec{g}) \cdot \vec{s} \mathrm{~d} s=0 \quad \forall \vec{s} \in \mathbf{H}^{-1 / 2}(\Gamma(\alpha))
\end{array}\right.
$$

and $\left(\widehat{u}_{t}, \widehat{p}_{t}, q_{t}, \alpha_{t}\right) \in \mathcal{A}_{a d}$ is the solution of

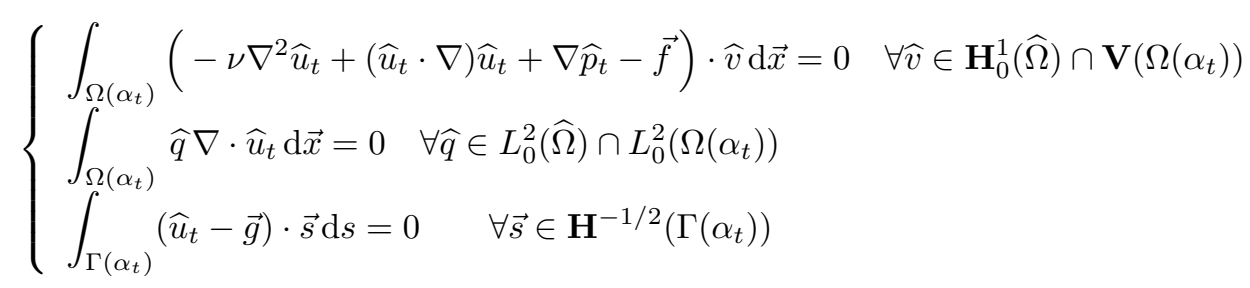

with $\vec{f}$ the body force defined over $\widehat{\Omega}$.

From the definition of Gateaux derivative we have to prove that

$$
\lim _{t \rightarrow 0+}\left(\frac{\left\|\widehat{u}_{t}-\widehat{u}-t \vec{u}^{\prime}\right\|_{\mathbf{H}^{1}(\Omega(\alpha))}}{t}\right)=0 \quad \lim _{t \rightarrow 0+}\left(\frac{\left\|\widehat{p}_{t}-\widehat{p}-t \vec{p}^{\prime}\right\|_{L^{2}(\Omega(\alpha))}}{t}\right)=0
$$

where $\left(\vec{u}^{\prime}, p^{\prime}\right)$ satisfies

$$
\left\{\begin{array}{l}
\int_{\Omega(\alpha)}\left(-\nu \nabla^{2} \vec{u}^{\prime}+\left(\vec{u}^{\prime} \cdot \nabla\right) \widehat{u}+(\widehat{u} \cdot \nabla) \vec{u}^{\prime}+\nabla p^{\prime}\right) \cdot \widehat{v} \mathrm{~d} \vec{x} \\
+\int_{\Gamma_{\alpha}}(\vec{V} \cdot \vec{n})\left(-\nu \nabla^{2} \widehat{u}+(\widehat{u} \cdot \nabla) \widehat{u}+\nabla \widehat{p}-\vec{f}\right) \cdot \widehat{v} \mathrm{~d} s=0 \quad \forall \widehat{v} \in \mathbf{H}_{0}^{1}(\widehat{\Omega}) \cap \mathbf{V}(\Omega(\alpha)) \\
\int_{\Omega(\alpha)} \widehat{q} \nabla \cdot \vec{u}^{\prime} \mathrm{d} \vec{x}+\int_{\Gamma_{\alpha}}(\vec{V} \cdot \vec{n}) \widehat{q} \nabla \cdot \widehat{u} \mathrm{~d} s=0 \quad \forall \widehat{q} \in L_{0}^{2}(\widehat{\Omega}) \cap L_{0}^{2}(\Omega(\alpha)) \\
\int_{\Gamma(\alpha)} \vec{u}^{\prime} \cdot \vec{s} \mathrm{~d} s+\int_{\Gamma_{\alpha}} \frac{\partial \widehat{u}}{\partial n}(\vec{V} \cdot \vec{n}) \cdot \vec{s} \mathrm{~d} s=0 \quad \forall \vec{s} \in \mathbf{H}^{-1 / 2}(\Gamma(\alpha)) .
\end{array}\right.
$$

It is clear that the formulation in (3.10) implies (3.6) when test functions vanishing at the boundary are used.

Now we write the equations for $\widetilde{u}=\left(\widehat{u}_{t}-\widehat{u}-t \vec{u}^{\prime}\right) / t$ and $\widetilde{p}=\left(\widehat{p}_{t}-\widehat{p}-t p^{\prime}\right) / t$ and show that $(\widetilde{u}, \widetilde{p})$ satisfies the limit in (3.9). By subtracting the first equation in (3.10) and (3.7) from (3.8) we can write

$$
\begin{aligned}
\int_{\Omega\left(\alpha_{t}\right)}( & \left.-\nu \nabla^{2} \widehat{u}_{t}+\left(\widehat{u}_{t} \cdot \nabla\right) \widehat{u}_{t}+\nabla \widehat{p}_{t}-\vec{f}\right) \cdot \widehat{v} \mathrm{~d} \vec{x}-\int_{\Omega(\alpha)}\left(-\nu \nabla^{2} \widehat{u}+(\widehat{u} \cdot \nabla) \widehat{u}+\nabla \widehat{p}-\vec{f}\right) \cdot \widehat{v} \mathrm{~d} \vec{x} \\
& -t \int_{\Omega(\alpha)}\left(-\nu \nabla^{2} \vec{u}^{\prime}+\left(\vec{u}^{\prime} \cdot \nabla\right) \widehat{u}+(\widehat{u} \cdot \nabla) \vec{u}^{\prime}+\nabla p^{\prime}\right) \cdot \widehat{v} \mathrm{~d} \vec{x} \\
& -t \int_{\Gamma_{\alpha}}(\vec{V} \cdot \vec{n})\left(-\nu \nabla^{2} \widehat{u}+(\widehat{u} \cdot \nabla) \widehat{u}+\nabla \widehat{p}-\vec{f}\right) \cdot \widehat{v} \mathrm{~d} s=0 .
\end{aligned}
$$


If we define $\widehat{w}=\widehat{u}_{t}-\widehat{u}, \widehat{z}=\widehat{p}_{t}-\widehat{p}$, and $F_{1}(\widehat{w}, \widehat{v})$ by

$$
\begin{aligned}
F_{1}(\widehat{w}, \widehat{v})= & -\frac{1}{t} \int_{\Omega\left(\alpha_{t}\right)}\left(-\nu \nabla^{2} \widehat{u}_{t}+\left(\widehat{u}_{t} \cdot \nabla\right) \widehat{u}_{t}+\nabla \widehat{p}_{t}-\vec{f}\right) \cdot \widehat{v} \mathrm{~d} \vec{x} \\
& +\frac{1}{t} \int_{\Omega(\alpha)}\left(-(\widehat{w} \cdot \nabla) \widehat{w}-\nu \nabla^{2} \widehat{u_{t}}+\left(\widehat{u_{t}} \cdot \nabla\right) \widehat{u}_{t}+\nabla \widehat{p}_{t}-\vec{f}\right) \cdot \widehat{v} \mathrm{~d} \vec{x} \\
& +\int_{\Gamma_{\alpha}}(\vec{V} \cdot \vec{n})\left(-\nu \nabla^{2} \widehat{u}+(\widehat{u} \cdot \nabla) \widehat{u}+\nabla \widehat{p}-\vec{f}\right) \cdot \widehat{v} \mathrm{~d} s
\end{aligned}
$$

for all $\widehat{v} \in \mathbf{V}(\Omega(\alpha)) \cap \mathbf{H}_{0}^{1}(\widehat{\Omega})$, then we can write

$$
\int_{\Omega(\alpha)}\left(-\nu \nabla^{2} \widetilde{u}+(\widetilde{u} \cdot \nabla) \widehat{u}+(\widehat{u} \cdot \nabla) \widetilde{u}+\nabla \widetilde{p}\right) \cdot \widehat{v} \mathrm{~d} \vec{x}=F_{1}(\widehat{w}, \widehat{v}) .
$$

In a similar way, we have

$$
\begin{aligned}
& \int_{\Omega(\alpha)} \widehat{q} \nabla \cdot \widetilde{u} \mathrm{~d} \vec{x}=F_{2}(\widehat{w}, \widehat{q}) \quad \forall \widehat{q} \in L_{0}^{2}(\widehat{\Omega}) \cap L_{0}^{2}(\Omega(\alpha)) \\
& \int_{\Gamma(\alpha)} \widetilde{u} \cdot \vec{s} \mathrm{~d} s=F_{3}(\widehat{w}, \vec{s}) \quad \forall \vec{s} \in \mathbf{H}^{-1 / 2}(\Gamma(\alpha))
\end{aligned}
$$

by defining

$$
F_{2}(\widehat{w}, \widehat{q})=-\frac{1}{t}\left(\int_{\Omega\left(\alpha_{t}\right)} \widehat{q} \nabla \cdot \widehat{u}_{t} \mathrm{~d} \vec{x}-\int_{\Omega(\alpha)} \widehat{q} \nabla \cdot \widehat{u}_{t} \mathrm{~d} \vec{x}\right)+\int_{\Gamma_{\alpha}}(\vec{V} \cdot \vec{n}) \widehat{q} \nabla \cdot \widehat{u} \mathrm{~d} s
$$

and

$$
F_{3}(\widehat{w}, \vec{s})=-\frac{1}{t}\left(\int_{\Gamma\left(\alpha_{t}\right)}\left(\widehat{u}_{t}-\vec{g}\right) \cdot \vec{s} \mathrm{~d} s-\int_{\Gamma(\alpha)}\left(\widehat{u}_{t}-\vec{g}\right) \cdot \vec{s} \mathrm{~d} s\right)+\int_{\Gamma_{\alpha}}(\vec{V} \cdot \vec{n}) \frac{\partial \widehat{u}}{\partial n} \cdot \vec{s} \mathrm{~d} s .
$$

Now, we use Lemma 3.1 to estimate $F_{1}, F_{2}$ and $F_{3}$. In fact, from Lemma 3.1, we have that for every $\epsilon \geq 0$ there exists a $t_{1}$ such that

$$
\begin{aligned}
\mid \frac{1}{t} \int_{\Omega\left(\alpha_{t}\right)}( & \left.-\nu \nabla^{2} \widehat{u}_{t}+\left(\widehat{u}_{t} \cdot \nabla\right) \widehat{u}_{t}+\nabla \widehat{p}_{t}-\vec{f}\right) \mathrm{d} \vec{x}-\frac{1}{t} \int_{\Omega(\alpha)}\left(-\nu \nabla^{2} \widehat{u}_{t}+\left(\widehat{u}_{t} \cdot \nabla\right) \widehat{u}_{t}+\nabla \widehat{p}_{t}-\vec{f}\right) \mathrm{d} \vec{x} \\
& -\int_{\Gamma_{\alpha}}(\vec{V} \cdot \vec{n})\left(-\nu \nabla^{2} \widehat{u}_{t}+\left(\widehat{u}_{t} \cdot \nabla\right) \widehat{u}_{t}+\nabla \widehat{p}_{t}-\vec{f}\right) \cdot \widehat{v} \mathrm{~d} s \mid \leq \epsilon
\end{aligned}
$$

for all $t<t_{1}$. Therefore, if $t<t_{1}$, we can write

$$
\left|F_{1}(\widehat{w}, \widehat{v})\right| \leq \epsilon+\left|\int_{\Gamma_{\alpha}} \vec{H}_{1}(\widehat{w}) \cdot \widehat{v} \mathrm{~d} s\right|+\left|\int_{\Omega(\alpha)} \vec{G}_{1}(\widehat{w}) \cdot \widehat{v} \mathrm{~d} \vec{x}\right|,
$$

where $\vec{H}_{1}(\widehat{w})$ and $\vec{G}_{1}(\widehat{w})$ are defined by

$$
\left.\int_{\Omega(\alpha)} \vec{G}_{1}(\widehat{w}) \cdot \widehat{v} \mathrm{~d} \vec{x}=\frac{1}{t} \int_{\Omega(\alpha)}((\widehat{w} \cdot \nabla) \widehat{w}) \cdot \widehat{v}\right) \mathrm{d} \vec{x}
$$

and

$$
\int_{\Gamma_{\alpha}} \vec{H}_{1}(\widehat{w}) \cdot \widehat{v} \mathrm{~d} s=\int_{\Gamma_{\alpha}}(\vec{V} \cdot \vec{n})\left(-\nu \nabla^{2} \widehat{w}+(\widehat{w} \cdot \nabla) \widehat{u}+(\widehat{u} \cdot \nabla) \widehat{w}+\nabla \widehat{z}\right) \cdot \widehat{v} \mathrm{~d} s
$$


for all $\widehat{v} \in \mathbf{H}_{0}^{1}(\widehat{\Omega}) \cap \mathbf{V}(\Omega(\alpha))$. Furthermore, for every $\epsilon>0$ there exists a $t_{2}$ such that for all $t<t_{2}$ we have

$$
\begin{aligned}
& \left|F_{2}(\widehat{w}, \widehat{v})\right| \leq \epsilon+\left|\int_{\Gamma_{\alpha}}(\vec{V} \cdot \vec{n}) \widehat{q} \nabla \cdot \widehat{w} \mathrm{~d} \vec{x}\right|=\epsilon+\left|\int_{\Gamma_{\alpha}} H_{2}(\widehat{w}) \widehat{q} \mathrm{~d} \vec{x}\right| \\
& \left|F_{3}(\widehat{w}, \vec{s})\right| \leq \epsilon+\left|\int_{\Gamma_{\alpha}}(\vec{V} \cdot \vec{n}) \frac{\partial \widehat{w}}{\partial n} \cdot \vec{s} \mathrm{~d} s\right|=\epsilon+\left|\int_{\Gamma_{\alpha}} \vec{H}_{3}(\widehat{w}) \cdot \vec{s} \mathrm{~d} s\right| .
\end{aligned}
$$

We note that the system (3.11)-(3.13) takes the form of a linearized Navier-Stokes system with the right-hand side terms given by $F_{1}, F_{2}$, and $F_{3}$. The norm of $\widetilde{u}$ and $\widetilde{p}$ can be evaluated by standard methods and bounded by the norm of the function $\vec{G}_{1}, \vec{H}_{1}, H_{2}$ and $\vec{H}_{3}$. We recall that the standard techniques require the condition (1.13) be satisfied and therefore (1.12) has a unique solution [37].

By using the trace theorem, the properties of the trilinear form $c(\widehat{w} ; \widehat{u}, \widehat{w})$, and standard arguments (see, e.g., [37]), the norms of $\vec{G}_{1}, \vec{H}_{1}, H_{2}$ and $\vec{H}_{3}$ can be bounded by the norm of $\widehat{w}$ and $\widehat{z}$. We shall show that these functions and therefore also the norm of $\widetilde{u}$ and $\widetilde{p}$ vanish when $t$ tends to zero which proves the theorem.

Now we need to evaluate the norm $\|\widehat{w}\|_{H^{1 / 2}(\Gamma(\alpha))},\|\nabla \widehat{w}\|,\|\nabla \widehat{z}\|$, and $\left\|\nabla^{2} \widehat{w}\right\|$ where the vector $\widehat{w}=\widehat{u}_{t}-\widehat{u}$ and $\widehat{z}=\widehat{p}_{t}-p$ are solutions of

$$
\begin{aligned}
\int_{\Omega(\alpha)}\left(-\nu \nabla^{2} \widehat{w}+(\widehat{w} \cdot \nabla) \widehat{u}+(\widehat{u} \cdot \nabla) \widehat{w}+\nabla \widehat{z}\right) \cdot \widehat{v} \mathrm{~d} \vec{x} & =F_{4}\left(\widehat{u}_{t}, \widehat{p}_{t}, \widehat{v}\right) \quad \forall \widehat{v} \in \mathbf{V}(\Omega(\alpha)) \cap \mathbf{H}_{0}^{1}(\widehat{\Omega}) \\
\int_{\Omega(\alpha)} \widehat{q} \nabla \cdot \widehat{w} \mathrm{~d} \vec{x} & =F_{5}\left(\widehat{u}_{t}, \widehat{p}_{t}, \widehat{q}\right) \quad \forall \widehat{q} \in L_{0}^{2}(\widehat{\Omega}) \cap L_{0}^{2}(\Omega(\alpha)) \\
\int_{\Gamma(\alpha)} \widehat{w} \cdot \vec{s} \mathrm{~d} s & =F_{6}\left(\widehat{u}_{t}, \widehat{p}_{t}, \vec{s}\right) \quad \forall \vec{s} \in \mathbf{H}^{-1 / 2}(\Gamma(\alpha))
\end{aligned}
$$

where

$$
\begin{aligned}
F_{4}\left(\widehat{u}_{t}, \widehat{p}_{t}, \widehat{v}\right)= & -\int_{\Omega\left(\alpha_{t}\right)}\left(-\nu \nabla^{2} \widehat{u}_{t}+\left(\widehat{u}_{t} \cdot \nabla\right) \widehat{u}_{t}+\nabla \widehat{p}_{t}-\vec{f}\right) \cdot \widehat{v} \mathrm{~d} \vec{x} \\
& +\int_{\Omega(\alpha)}\left(-(\widehat{w} \cdot \nabla) \widehat{w}-\nu \nabla^{2} \widehat{u}_{t}+\left(\widehat{u}_{t} \cdot \nabla\right) \widehat{u}_{t}+\nabla \widehat{p}_{t}-\vec{f}\right) \cdot \widehat{v} \mathrm{~d} \vec{x}, \\
F_{5}\left(\widehat{u}_{t}, \widehat{p}_{t}, \widehat{q}\right)= & -\int_{\Omega\left(\alpha_{t}\right)} \widehat{q} \nabla \cdot \widehat{u}_{t} \mathrm{~d} \vec{x}+\int_{\Omega(\alpha)} \widehat{q} \nabla \cdot \widehat{u}_{t} \mathrm{~d} \vec{x},
\end{aligned}
$$

and

$$
F_{6}\left(\widehat{u}_{t}, \widehat{p}_{t}, \vec{s}\right)=-\int_{\Gamma\left(\alpha_{t}\right)}\left(\widehat{u}_{t}-\vec{g}\right) \cdot \vec{s} \mathrm{~d} s+\int_{\Gamma(\alpha)}\left(\widehat{u}_{t}-\vec{g}\right) \cdot \vec{s} \mathrm{~d} s .
$$

Again, we use Lemma 3.1 to estimate $F_{4}, F_{5}$, and $F_{6}$. It is easy to see that for all $\epsilon>0$ there is a $t_{3}$ such that for all $t \leq t_{3}$ we have

$$
\begin{aligned}
& \left|F_{4}\right| \leq \frac{\epsilon}{2}+\left|\int_{\Omega(\alpha)} \vec{G}_{4} \cdot \widehat{v} \mathrm{~d} \vec{x}\right| \quad \forall \widehat{v} \in \mathbf{V}(\Omega(\alpha)) \cap \mathbf{H}_{0}^{1}(\widehat{\Omega}) \\
& \left|F_{5}\right| \leq \frac{\epsilon}{2}+\left|\int_{\Omega(\alpha)} G_{5} \widehat{q} \mathrm{~d} \vec{x}\right| \quad \forall \widehat{q} \in L_{0}^{2}(\widehat{\Omega}) \cap L_{0}^{2}(\Omega(\alpha)) \\
& \left|F_{6}\right| \leq \frac{\epsilon}{2}+\left|\int_{\Gamma(\alpha)} \vec{H}_{6} \cdot \vec{s} \mathrm{~d} \vec{x}\right| \quad \forall \vec{s} \in \mathbf{H}^{-1 / 2}(\Gamma(\alpha))
\end{aligned}
$$

with $\left\|\vec{G}_{4}\right\|_{L^{2}},\left\|G_{5}\right\|_{L^{2}}$ and $\left\|\vec{H}_{6}\right\|_{H^{1 / 2}}$ bounded by $\epsilon / 2$. 
Since $\epsilon$ is positive but arbitrarily small, the set (3.18)-(3.20) is a linearized version of the Navier-Stokes system with regular and arbitrarily small right-hand side. Again, if the condition (1.13) is satisfied (so that (1.12) has a unique solution), by using standard arguments [37], we find that for all $\delta>0$ there is a $t_{4}$ such that, for all $t<t_{4}$, the norms of the solution $(\widehat{w}, \widehat{p})$ are bounded by $\delta$. From standard regularity results we can conclude that $\|\widehat{w}\|_{H^{1 / 2}},\|\widehat{w}\|_{2}$, and $\|\widehat{p}\|_{1}$ can be bounded by arbitrarily small positive constants when $t$ tends to zero.

Under the hypotheses of the Theorem 3.2, we have the existence of the Gateaux derivative of the map $(\vec{u}, p)=(\vec{u}(\alpha), p(\alpha))$. Clearly, the map $q=q(\alpha)$ from $\mathcal{Q}_{a d}$ to $L^{2}(I)$ is differentiable at $\alpha \in \mathcal{Q}_{a d}$ in the direction $\delta \alpha$ and its Gateaux derivative $q^{\prime}=D q / D \alpha \cdot \delta \alpha$ satisfies the following equation

$$
\int_{I} v q^{\prime} \mathrm{d} x+\int_{I} \frac{\mathrm{d} v}{\mathrm{~d} x} \frac{\mathrm{d} \delta \alpha}{\mathrm{d} x} \mathrm{~d} x=0 \quad \forall v \in H_{0}^{1}(I)
$$

Now, it is easy to show the differentiability of the functional $\mathcal{J}(\vec{u}, q, \alpha)$.

Theorem 3.3. Let the hypotheses of the Theorem 3.2 hold. Let $(\vec{u}, p, q, \alpha) \in \mathcal{A}_{a d}$ have Gateaux derivative $\vec{u}^{\prime} \in \mathbf{H}^{1}(\Omega(\alpha))$ satisfying (3.6). Let $q^{\prime} \in L^{2}(I)$ satisfy (3.21). Then, the functional in (1.7) defines a mapping

$$
\mathcal{J}(\vec{u}(\alpha), q(\alpha), \alpha): \mathcal{Q}_{a d} \rightarrow \mathbb{R}
$$

This mapping has a Gateaux derivative $\mathcal{J}^{\prime}(\vec{u}, q, \alpha)$ in the direction $\delta \alpha=\widetilde{\alpha}-\alpha$ for all admissible $\widetilde{\alpha}$. Furthermore, we have

$$
\mathcal{J}^{\prime}(\vec{u}(\alpha), q(\alpha), \alpha)=\frac{D \mathcal{J}(\vec{u}, q, \alpha)}{D \alpha} \cdot \delta \alpha=2 \nu \int_{\Omega(\alpha)} \nabla \vec{u} \cdot \nabla \vec{u}^{\prime} \mathrm{d} \vec{x}+\nu \int_{\Gamma_{\alpha}} \frac{\partial \vec{u}}{\partial n} \frac{\partial \vec{u}}{\partial n}(\vec{V} \cdot \vec{n}) \mathrm{d} s+\beta \int_{I} q q^{\prime} \mathrm{d} x
$$

where $\vec{V}$ is defined by $(0, \delta \alpha)$ on $\Gamma_{\alpha}$ and $\vec{n}$ the unit vector normal to the boundary.

Proof. The results are a consequence of Theorem 3.2 and Lemma 3.1. Let $\alpha$ and $\widetilde{\alpha}$ be given in $\mathcal{Q}_{a d}$. We set $\delta \alpha=\widetilde{\alpha}-\alpha$ and $\alpha_{t}=\alpha+t \delta \alpha$. We can write

$$
\begin{aligned}
\lim _{t \rightarrow 0} \frac{\nu}{t}\left(\int_{\Omega\left(\alpha_{t}\right)} \nabla \vec{u}_{t} \cdot \nabla \vec{u}_{t} \mathrm{~d} \vec{x}-\int_{\Omega(\alpha)} \nabla \vec{u} \cdot \nabla \vec{u} \mathrm{~d} \vec{x}\right)= & \lim _{t \rightarrow 0} \frac{\nu}{t}\left(\int_{\Omega\left(\alpha_{t}\right)} \nabla \vec{u}_{t} \cdot \nabla \vec{u}_{t} \mathrm{~d} \vec{x}-\int_{\Omega(\alpha)} \nabla \vec{u}_{t} \cdot \nabla \vec{u}_{t} \mathrm{~d} \vec{x}\right) \\
& +\lim _{t \rightarrow 0} \frac{\nu}{t}\left(\int_{\Omega(\alpha)} \nabla \vec{u}_{t} \cdot \nabla \vec{u}_{t} \mathrm{~d} \vec{x}-\int_{\Omega(\alpha)} \nabla \vec{u} \cdot \nabla \vec{u} \mathrm{~d} \vec{x}\right)
\end{aligned}
$$

which converges, from Theorem 3.2 and Lemma 3.1, to

$$
2 \nu \int_{\Omega(\alpha)} \nabla \vec{u} \cdot \nabla \vec{u}^{\prime} \mathrm{d} \vec{x}+\nu \int_{\Gamma_{\alpha}}(\nabla \vec{u} \cdot \nabla \vec{u})(\vec{V} \cdot \vec{n}) \mathrm{d} s
$$

as $t$ tends to zero. The theorem follows by writing

$$
\int_{\Gamma_{\alpha}}(\nabla \vec{u} \cdot \nabla \vec{u})(\vec{V} \cdot \vec{n}) \mathrm{d} s=\int_{\Gamma_{\alpha}} \frac{\partial \vec{u}}{\partial n} \frac{\partial \vec{u}}{\partial n}(\vec{V} \cdot \vec{n}) \mathrm{d} s
$$

since $\vec{u}=\overrightarrow{0}$ on $\Gamma_{\alpha}$. The derivative of the term involving $q$ is obvious and this completes the proof. 


\subsection{The optimality system}

We show that the optimal control problem implies a first-order necessary condition. If $(\vec{u}, p, q, \alpha)$ is an optimal solution, then for every $\delta \alpha=\widetilde{\alpha}-\alpha$ such that $\widetilde{\alpha} \in \mathcal{Q}_{a d}$ and for every $\lambda \in \mathbb{R}^{+}$, we have, from the definition of an optimal solution,

$$
\mathcal{J}(\vec{u}(\alpha+\lambda \delta \widetilde{\alpha}), q(\alpha+\lambda \delta \widetilde{\alpha}), \alpha+\lambda \delta \widetilde{\alpha}) \geq \mathcal{J}(\vec{u}, q, \alpha)
$$

The above inequality implies

$$
\frac{\mathcal{J}(\vec{u}(\alpha+\lambda \delta \widetilde{\alpha}), q(\alpha+\lambda \delta \widetilde{\alpha}), \alpha+\lambda \delta \widetilde{\alpha})-\mathcal{J}(\vec{u}, q, \alpha)}{\lambda} \geq 0 \quad \text { if } \quad \lambda \geq 0 .
$$

The limit must be non-negative when $\lambda$ tends to zero and this leads to the following first-order necessary condition.

Theorem 3.4. If $(\vec{u}, p, q, \alpha)$ is an optimal pair for the problem in (2.2) and the functional in (1.7) is Gateaux differentiable, then the necessary condition for $\alpha$ to be a minimizer is

$$
\mathcal{J}^{\prime}(\vec{u}, q, \widetilde{\alpha}-\alpha) \geq 0
$$

for all $\widetilde{\alpha} \in \mathcal{Q}_{a d}$ such that $\widetilde{\alpha}^{\prime \prime} \in \mathcal{B}_{a d}$.

We recall that the set of all $\widetilde{\alpha} \in \mathcal{Q}_{a d}$ such that $\widetilde{\alpha}^{\prime \prime} \in \mathcal{B}_{a d}$ is a convex closed set and is not empty from the definition of $\mathcal{B}_{a d}$.

In Theorem 3.7, we shall show that the condition (3.24) can be written explicitly and the solution of the problem in (2.2) satisfies a system of equations and variational inequalities. In order to do this, we will need the following interesting preliminary results.

Lemma 3.5. Let $\alpha$ and $\widetilde{\alpha}$ be in $\mathcal{Q}_{a d}$ with $\delta \alpha=\widetilde{\alpha}-\alpha$ and $\vec{u}^{\prime}$ be defined by (3.6). Then, for every $\widetilde{h}_{2}$ in $\mathbf{H}^{1}(\Omega(\alpha))$, we have

$$
\int_{\Omega(\alpha)} \nabla \widetilde{h}_{2} \cdot \nabla \vec{u}^{\prime} \mathrm{d} \vec{x}=-\nu \int_{\Gamma_{\alpha}} \frac{\partial \vec{u}}{\partial n} \frac{\partial \vec{w}}{\partial n}(\vec{V} \cdot \vec{n}) \mathrm{d} s
$$

where $\vec{V}=(0, \delta \alpha)$ is defined on $\Gamma_{\alpha}$ and $\vec{w}$ is the solution of the linear adjoint problem

$$
\left\{\begin{array}{l}
\nu a(\vec{w}, \vec{v})+c(\vec{v} ; \vec{u}, \vec{w})+c(\vec{u} ; \vec{v}, \vec{w})+b(\vec{v}, \sigma)=-\int_{\Omega(\alpha)} \nabla \widetilde{h}_{2} \cdot \nabla \vec{v} \mathrm{~d} \vec{x} \quad \forall \vec{v} \in \mathbf{H}_{0}^{1}(\Omega(\alpha)) \\
b(\vec{w}, q)=0 \quad \forall q \in L_{0}^{2}(\Omega(\alpha)) \\
\langle\vec{w}, \vec{s}\rangle_{\Gamma(\alpha)}=0 \quad \forall \vec{s} \in \mathbf{H}^{-1 / 2}(\Gamma(\alpha)) .
\end{array}\right.
$$

Proof. If we write the first equation in (3.25) with test functions in $\mathbf{H}^{1}(\Omega(\alpha))$, we have

$\nu a(\vec{w}, \vec{v})+c(\vec{v} ; \vec{u}, \vec{w})+c(\vec{u} ; \vec{v}, \vec{w})+b(\vec{v}, \sigma)+\int_{\Gamma(\alpha)}\left(\sigma \vec{n}-\nu \frac{\partial \vec{w}}{\partial n}\right) \cdot \vec{v} \mathrm{~d} s=-\int_{\Omega(\alpha)} \nabla \widetilde{h}_{2} \cdot \nabla \vec{v} \mathrm{~d} \vec{x} . \quad \forall \vec{v} \in \mathbf{H}^{1}(\Omega(\alpha))$.

By using this equation with $\vec{v}=\vec{u}^{\prime}$ and $q=p^{\prime}$, we can write

$$
-\int_{\Omega(\alpha)} \nabla \widetilde{h}_{2} \cdot \nabla \vec{u}^{\prime} \mathrm{d} \vec{x}=\int_{\Gamma(\alpha)}\left(\sigma \vec{n}-\nu \frac{\partial \vec{w}}{\partial n}\right) \cdot \vec{u}^{\prime} \mathrm{d} s+\nu a\left(\vec{w}, \vec{u}^{\prime}\right)+c\left(\vec{u}^{\prime} ; \vec{u}, \vec{w}\right)+c\left(\vec{u} ; \vec{u}^{\prime}, \vec{w}\right)+b\left(\vec{u}^{\prime}, \sigma\right) .
$$


The result follows from (3.6), the weak equation for the Gateaux derivative, with $\vec{v}=\vec{w}$ and from $b\left(\vec{w}, p^{\prime}\right)=0$. In fact

$$
\begin{aligned}
\nu a\left(\vec{u}^{\prime}, \vec{w}\right)+c\left(\vec{u}^{\prime} ; \vec{u} ; \vec{w}\right)+c\left(\vec{u} ; \vec{u}^{\prime}, \vec{w}\right)-\int_{\Gamma_{\alpha}}\left(\sigma \vec{n}-\nu \frac{\partial \vec{w}}{\partial n}\right) \cdot \frac{\partial \vec{u}}{\partial n}(\vec{V} \cdot \vec{n}) \mathrm{d} s & =\int_{\Gamma_{\alpha}}\left(-\sigma \vec{n}+\nu \frac{\partial \vec{w}}{\partial n}\right) \frac{\partial \vec{u}}{\partial n}(\vec{V} \cdot \vec{n}) \mathrm{d} s \\
& =\int_{\Gamma_{\alpha}} \nu \frac{\partial \vec{w}}{\partial n} \frac{\partial \vec{u}}{\partial n}(\vec{V} \cdot \vec{n}) \mathrm{d} s
\end{aligned}
$$

since $\vec{n} \cdot \frac{\partial \vec{u}}{\partial n}=\frac{\partial u_{s}}{\partial s}=0$ (see [29]).

Lemma 3.6. Let $\alpha$ and $\widetilde{\alpha}$ be in $\mathcal{Q}_{a d}$ with $\delta \alpha=\widetilde{\alpha}-\alpha$ and $q^{\prime}$ be defined by (3.21). Then, for every $q$ in $L^{2}(I)$, we have

$$
\beta \int_{I} q q^{\prime} \mathrm{d} x=\int_{I} \frac{d \mu}{\mathrm{d} x} \frac{\mathrm{d} \delta \alpha}{\mathrm{d} x} \mathrm{~d} x
$$

where $\mu$ is the solution of the linear equation

$$
\int_{I}(\mu+\beta q) \phi \mathrm{d} x=0 \quad \forall \phi \in L^{2}(I) .
$$

Proof. The proof follows easily by combining (3.21), (3.27), and the boundary conditions for $\alpha$.

We now write out conditions (3.24) explicitly, using the solution of an adjoint system.

Theorem 3.7. If $(\vec{u}, p, q, \alpha)$ is optimal for the problem (2.2), satisfying the hypotheses of Theorem 3.2, then $\alpha$ is solution of

$$
\mathcal{J}^{\prime}(\vec{u}, q, \alpha)(\vec{w}, \mu, \widetilde{\alpha}-\alpha) \geq 0 \quad \forall \widetilde{\alpha} \in \mathcal{Q}_{a d}
$$

where

$$
\mathcal{J}^{\prime}(\vec{u}, q, \alpha)(\vec{w}, \mu, \zeta)=\nu \int_{\Gamma_{\alpha}}\left(\frac{\partial \vec{u}}{\partial n} \frac{\partial \vec{u}}{\partial n}-\frac{\partial \vec{u}}{\partial n} \frac{\partial \vec{w}}{\partial n}\right)(\vec{V}(\zeta) \cdot \vec{n}) \mathrm{d} s+\int_{I} \frac{\mathrm{d} \mu}{\mathrm{d} x} \frac{\mathrm{d} \zeta}{\mathrm{d} x} \mathrm{~d} x
$$

The vector $\vec{V}(\zeta)$ is simply $(0, \zeta), \vec{n}$ is the unit normal to $\Gamma_{\alpha}$, and $\mu+\beta q=0$, where $q \in \mathcal{B}_{a d}$ and the function $\vec{w}$ is solution of the adjoint system

$$
\left\{\begin{array}{l}
\nu a(\vec{w}, \vec{v})+c(\vec{v} ; \vec{u}, \vec{w})+c(\vec{u} ; \vec{v}, \vec{w})+b(\vec{v}, \sigma)=-2 \nu \int_{\Omega(\alpha)}(\nabla \vec{u} \cdot \nabla \vec{v}) \mathrm{d} \vec{x} \quad \forall \vec{v} \in \mathbf{H}_{0}^{1}(\Omega(\alpha)) \\
b(\vec{w}, q)=0 \quad \forall q \in L_{0}^{2}(\Omega(\alpha)) \\
\vec{w}=\overrightarrow{0} \quad \forall \vec{x} \in \Gamma(\alpha) .
\end{array}\right.
$$


Proof. Let $(\vec{u}, p, q, \alpha)$ be an optimal solution of the problem (2.2). By Theorem 3.3 we compute the Gateaux derivative of the functional $\mathcal{J}(\vec{u}, q, \alpha)$ and then Lemma 3.5 completes the proof. We have

$$
\mathcal{J}^{\prime}(\vec{u}(\alpha), q(\alpha), \alpha)=\frac{D \mathcal{J}(\vec{u}, q, \alpha)}{D \alpha} \cdot \delta \alpha=2 \nu \int_{\Omega(\alpha)} \nabla \vec{u} \cdot \nabla \vec{u}^{\prime} \mathrm{d} \vec{x}+\nu \int_{\Gamma_{\alpha}} \frac{\partial \vec{u}}{\partial n} \frac{\partial \vec{u}}{\partial n}(\vec{V} \cdot \vec{n}) \mathrm{d} s+\beta \int_{I} q q^{\prime} \mathrm{d} x
$$

where $\vec{u}^{\prime}$ is a solution of $(3.6), q^{\prime}$ solution of $(3.21), \vec{V}$ defined by $(0, \zeta)$ on $\Gamma_{\alpha}$ and $\vec{n}$ the normal unit vector. Now, by using Lemmas 3.5 and 3.6, we obtain

$$
\mathcal{J}^{\prime}(\vec{u}, q, \alpha)(\vec{w}, \mu, \delta \alpha)=\int_{I} \frac{\mathrm{d} \mu}{\mathrm{d} x} \frac{\mathrm{d} \delta \alpha}{\mathrm{d} x} \mathrm{~d} x+\nu \int_{\Gamma_{\alpha}}\left(\frac{\partial \vec{u}}{\partial n} \frac{\partial \vec{u}}{\partial n}-\frac{\partial \vec{u}}{\partial n} \frac{\partial \vec{w}}{\partial n}\right)(\vec{V}(\delta \alpha) \cdot \vec{n}) \mathrm{d} s
$$

where $\vec{w}$ is the solution of (3.30). Now, the theorem follows from Theorem 3.4 and $\zeta=\delta \alpha=\widetilde{\alpha}-\alpha$.

To summarize, in order to solve the optimal problem for $(\vec{u}, \vec{w}, p, \sigma, q, \mu, \alpha)$ by using the adjoint equation method, we have to solve the Navier-Stokes system

$$
\left\{\begin{array}{l}
\nu a(\vec{u}, \vec{v})+c(\vec{u} ; \vec{u}, \vec{v})+b(\vec{v}, p)=\langle\vec{f}, \vec{v}\rangle \quad \forall \vec{v} \in \mathbf{H}_{0}^{1}(\Omega(\alpha)) \\
b(\vec{u}, q)=0 \quad \forall q \in L_{0}^{2}(\Omega(\alpha)) \\
\langle\vec{u}, \vec{s}\rangle_{\Gamma(\alpha)}=\langle\vec{g}, \vec{s}\rangle_{\Gamma(\alpha)} \quad \forall \vec{s} \in \mathbf{H}^{-1 / 2}(\Gamma(\alpha))
\end{array}\right.
$$

the adjoint system

$$
\left\{\begin{array}{l}
\nu a(\vec{w}, \vec{v})+c(\vec{w} ; \vec{u}, \vec{v})+c(\vec{u} ; \vec{w}, \vec{v})+b(\vec{v}, \sigma)=-2 \nu \int_{\Omega(\alpha)} \nabla \vec{u} \cdot \nabla \vec{v} \mathrm{~d} \vec{x} \quad \forall \vec{v} \in \mathbf{H}_{0}^{1}(\Omega(\alpha)) \\
b(\vec{w}, q)=0 \quad \forall q \in L_{0}^{2}(\Omega(\alpha)) \\
\langle\vec{w}, \vec{s}\rangle_{\Gamma(\alpha)}=0 \quad \forall \vec{s} \in \mathbf{H}^{-1 / 2}(\Gamma(\alpha)),
\end{array}\right.
$$

and the inequality

$$
\mathcal{L}^{\prime}(\vec{u}, \alpha)(\vec{w}, \widetilde{\alpha}-\alpha) \geq 0
$$

for all $\widetilde{\alpha} \in \mathcal{C}_{a d}$. The function $\mathcal{L}^{\prime}(\vec{u}, \alpha)(\vec{w}, \zeta)$ is defined by

$$
\mathcal{L}^{\prime}(\vec{u}, \alpha)(\vec{w}, \zeta)=\beta \int_{I} \frac{\mathrm{d}^{2} \alpha}{\mathrm{d} x^{2}} \frac{\mathrm{d}^{2} \zeta}{\mathrm{d} x^{2}} \mathrm{~d} x+\nu \int_{\Gamma_{\alpha}}\left(\frac{\partial \vec{u}}{\partial n} \frac{\partial \vec{u}}{\partial n}-\frac{\partial \vec{u}}{\partial n} \frac{\partial \vec{w}}{\partial n}\right)(\vec{V}(\zeta) \cdot \vec{n}) \mathrm{d} s
$$

with boundary conditions $\alpha(a)=z_{1}, \alpha(b)=z_{2}$, and $\alpha^{\prime}(a)=\alpha^{\prime}(b)=0$. The quantities $q$ and $\mu$ can be computed through

$$
\int_{I} q v \mathrm{~d} x=-\int_{I} \frac{\mathrm{d} \alpha}{\mathrm{d} x} \frac{\mathrm{d} v}{\mathrm{~d} x} \mathrm{~d} x \quad \forall v \in H_{0}^{1}(I)
$$

and $\mu+\beta q=0$, respectively. 


\subsection{The shape gradient}

We now have two methods for computing the shape gradient. Given a direction $\delta \alpha$ and a candidate optimizer $(\vec{u}, p, q, \alpha)$, we have that the shape gradient is given in terms of the sensitivity variables by (3.23), where $\vec{V}=(0, \delta \alpha)$ and where the flow sensitivity $\vec{u}^{\prime}$ is determined from (3.6). Alternately, the shape gradient is given in terms of adjoint variables by (3.29), where the adjoint variables $\vec{w}$ and $\mu$ are determined from (3.30) and $\mu+\beta q=0$, respectively.

It is important to note that the sensitivity equations (3.6) involve $\delta \alpha$ through the appearance of $\vec{V}$. Thus, if one changes the direction $\delta \alpha$, one must recompute the sensitivities. On the other hand, the adjoint system (3.30) is independent of the choice of $\delta \alpha$. This can have a profound effect on the cost of determining optimal solutions through methods that use the shape gradient. For any candidate optimizer $(\vec{u}, p, q, \alpha)$, the shape gradient can be computed for multiple directions $\delta \alpha$ with a single linear adjoint system solution. On the other hand, the sensitivity equation must be solved for each distinct direction $\delta \alpha$.

Of course, flow sensitivities are of interest in their own right. They describe how and where changes in the parameters that determine the shape of the boundary affect the flow. However, in the optimal control setting, it seems that using the adjoint equation approach to determining the shape gradient is more efficient.

\section{The Lagrange MUltiplier Method}

\subsection{Preliminaries}

In this section, we show that the Lagrange multiplier technique is well posed and can be used to obtain the first-order necessary condition. Further, the Lagrangian map can be shown to be strictly differentiable for all values of the external force and this allows us to apply the Lagrange multiplier method to a wider range of problems and completes the theoretical treatment of the problem for arbitrary values of the viscosity. Also, this method gives a different and better theoretical insight into the control process, allowing us to write the inequality constraints in a different form.

First, we introduce auxiliary variables that allow us to transform the inequality constraints into equalities and then invoke well-known derivations for equality constrained minimization problems; see, e.g., [3] or [39].

We begin by replacing

$$
c_{0} \leq \alpha \leq d_{0} \quad \text { and } \quad c_{2} \leq q \leq d_{2} \quad \forall x \in I
$$

by

$$
\begin{array}{rlrl}
\left|\alpha-\alpha_{0}\right|^{2}-\alpha_{m}^{2}+s_{0}^{2} & =0 & & \forall x \in I \\
\left|q-q_{0}\right|^{2}-q_{m}^{2}+s_{2}^{2} & =0 & \forall x \in I
\end{array}
$$

for some $s_{2} \in L^{2}(I)$ and $s_{0} \in H^{2}(I)$, where $\alpha_{0}=\left(c_{0}+d_{0}\right) / 2, q_{0}=\left(c_{2}+d_{2}\right) / 2, \alpha_{m}=\left(d_{0}-c_{0}\right) / 2$, and $q_{m}=\left(d_{2}-c_{2}\right) / 2$. Clearly, if (4.2)-(4.3) are satisfied, then so are (4.1). Also, note that if $(\vec{u}, p, q, \alpha)$ is a solution of the optimal control problem, then there exist $s_{0}, s_{2}$ such that $\alpha, q$ and $s_{0}, s_{2}$ satisfy (4.2)-(4.3).

In this section, we let $\Gamma(\alpha)$ be piecewise $C^{1,1}$ in agreement with the proposed model problem and $\vec{g} \in$ $\mathbf{H}^{3 / 2}(\Gamma(\alpha))$ where $\int_{\Gamma(\alpha)} \vec{g} \cdot \vec{n} \mathrm{~d} s=0$ with $\vec{g}=\overrightarrow{0}$ on $\Gamma_{2} \cap \Gamma_{4}, \vec{g}=\vec{g}_{1} \in \mathbf{H}^{3 / 2}\left(\Gamma_{1}\right)$ on $\Gamma_{1}$ and $\vec{g}=\vec{g}_{2} \in \mathbf{H}^{3 / 2}\left(\Gamma_{2}\right)$ on $\Gamma_{2}$.

The Lagrange multiplier method proposed here is based on an embedded technique method. We write the Lagrangian on a larger domain and then choose the solution that fits our domain forcing the boundary values as a constraint. 
Let $\widehat{\Omega}$ be an open bounded domain with boundary $\widehat{\Gamma} \in C^{1,1}$ with convex corners which contains $\Omega(\alpha)$ for all $\alpha$. We use the "hat notation" for functions on $\widehat{\Omega}$. Also let $\widehat{f} \in L^{2}(\widehat{\Omega})$ be the body force on the extended domain as previously discussed.

Let $\widehat{\mathbf{B}}_{1}=\left(\mathbf{H}^{2}(\widehat{\Omega}) \cap \mathbf{H}_{0}^{1}(\widehat{\Omega})\right) \times\left(L_{0}^{2}(\widehat{\Omega}) \cap \mathbf{H}^{1}(\widehat{\Omega})\right) \times \mathcal{B}_{a d} \times \mathcal{Q}_{a d} \times H^{2}(I) \times L^{2}(I), \widehat{\mathbf{B}}_{2}=\mathbf{H}^{-1}(\widehat{\Omega}) \times L_{0}^{2}(\widehat{\Omega}) \times$ $\mathbf{H}^{1 / 2}(I) \times \mathbf{H}^{1 / 2}\left(\Gamma(\alpha)-\Gamma_{\alpha}\right) \times L^{2}(I) \times H^{2}(I) \times L^{1}(I)$ and $\widehat{\mathbf{B}}_{3}=\mathbf{H}^{-1}(\widehat{\Omega}) \times L_{0}^{2}(\widehat{\Omega}) \times \mathbf{H}^{1 / 2}(I) \times \mathbf{H}^{1 / 2}\left(\Gamma(\alpha)-\Gamma_{\alpha}\right) \times$ $L^{2}(I) \times W^{2,1}(I) \times L^{1}(I)$. We equip $\widehat{\mathbf{B}}_{1}, \widehat{\mathbf{B}}_{2}$ and $\widehat{\mathbf{B}}_{3}$ with the usual graph norms for the product spaces involved.

We define the nonlinear mapping $M: \widehat{\mathbf{B}}_{1} \rightarrow \widehat{\mathbf{B}}_{3}$ by $M\left(\widehat{u}, \widehat{p}, q, \alpha, s_{0}, s_{2}\right)=\left(\vec{l}_{1}, l_{2}, \vec{l}_{3}, \vec{l}_{4}, l_{5}, l_{6}, l_{7}\right)$ for $\left(\widehat{u}, \widehat{p}, q, \alpha, s_{0}, s_{2}\right) \in \widehat{\mathbf{B}}_{1}$ and $\left(\vec{l}_{1}, l_{2}, \vec{l}_{3}, \vec{l}_{4}, l_{5}, l_{6}, l_{7}\right) \in \widehat{\mathbf{B}}_{3}$ if and only if

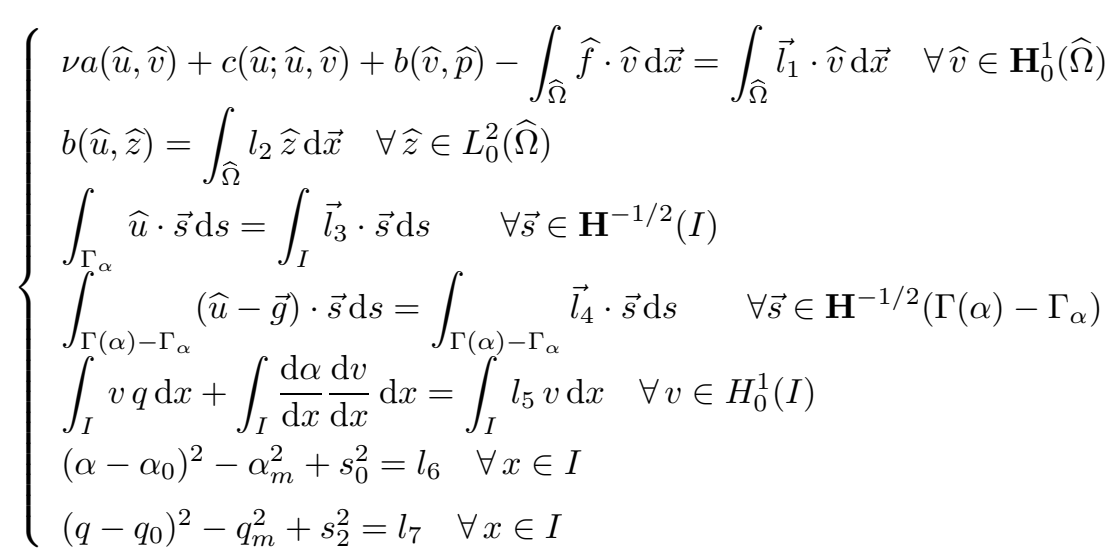

with $\alpha(a)=z_{1}, \alpha(b)=z_{2}$, and $\alpha^{\prime}(a)=\alpha^{\prime}(b)=0$. The set of constraint equations in the optimal shape control problem can be expressed as $M\left(\widehat{u}, \widehat{p}, q, \alpha, s_{0}, s_{2}\right)=(\overrightarrow{0}, 0, \overrightarrow{0}, \overrightarrow{0}, 0,0,0)$ when the test functions $\widehat{v}, \widehat{z}$ vanish outside $\widehat{\Omega}$.

Given $\left(\vec{u}_{1}, p_{1}, q_{1}, \alpha_{1}\right) \in \mathcal{A}_{a d}$, we define another nonlinear mapping $Q: \widehat{\mathbf{B}}_{1} \rightarrow \mathbb{R} \times \widehat{\mathbf{B}}_{3}$ by $Q\left(\widehat{u}, \widehat{p}, q, \alpha, s_{0}, s_{2}\right)=$ $\left(a, \vec{l}_{1}, l_{2}, \vec{l}_{3}, \vec{l}_{4}, l_{5}, l_{6}, l_{7}\right)$ if and only if

$$
\left(\begin{array}{c}
\mathcal{J}(\widehat{u}, q, \alpha)-\mathcal{J}\left(\vec{u}_{1}, q_{1}, \alpha_{1}\right) \\
M\left(\widehat{u}, \widehat{p}, q, \alpha, s_{0}, s_{2}\right)
\end{array}\right)=\left(\begin{array}{c}
a \\
\left(\vec{l}_{1}, l_{2}, \vec{l}_{3}, \vec{l}_{4}, l_{5}, l_{6}, l_{7}\right)
\end{array}\right)
$$

\subsection{Differentiability}

These mappings are strictly differentiable, as is shown in the following lemma. We recall the notion of strict differentiability (see [39]). Let $X$ and $Y$ denote Banach spaces, then the mapping $\varphi: X \rightarrow Y$ is strictly differentiable at $x \in X$ if there exists a bounded, linear mapping $D$ from $X$ to $Y$ such that for any $\epsilon>0$ there exists a $\delta>0$ such that whenever $\left\|x-x_{1}\right\|_{X}<\delta$ and $\left\|x-x_{2}\right\|_{X}<\delta$ for $x_{1}, x_{2} \in X$, then

$$
\left\|\varphi\left(x_{1}\right)-\varphi\left(x_{2}\right)-D\left(x_{1}-x_{2}\right)\right\|_{Y} \leq \epsilon\left\|x_{1}-x_{2}\right\|_{X}
$$


The strict derivative $D$ at the point $x \in X$, if it exists, will often be denoted by $D=\varphi^{\prime}(x)$. The value of this mapping on an element $\widetilde{x} \in X$ will often be denoted by $\varphi^{\prime}(x) \cdot \widetilde{x}$. In the next theorem we can identify $X=\widehat{\mathbf{B}}_{1}$ and $Y=\widehat{\mathbf{B}}_{2}$.

Lemma 4.1. Let the nonlinear mappings $M: \widehat{\mathbf{B}}_{1} \rightarrow \widehat{\mathbf{B}}_{3}$ and $Q: \widehat{\mathbf{B}}_{1} \rightarrow \mathbb{R} \times \widehat{\mathbf{B}}_{3}$ be defined by (4.4) and (4.5), respectively. Then, these mappings are strictly differentiable at a point $\left(\widehat{u}, \widehat{p}, q, \alpha, s_{0}, s_{2}\right) \in \widehat{\mathbf{B}}_{1}$ and its strict derivative is given by the bounded linear operator $M^{\prime}\left(\widehat{u}, \widehat{p}, q, \alpha, s_{0}, s_{2}\right): \widehat{\mathbf{B}}_{1} \rightarrow \widehat{\mathbf{B}}_{2}$, where $M^{\prime}\left(\widehat{u}, \widehat{p}, q, \alpha, s_{0}, s_{2}\right)$. $\left(\widetilde{u}, \widetilde{p}, \widetilde{q}, \widetilde{\alpha}, \widetilde{s}_{0}, \widetilde{s}_{2}\right)=\left(\bar{l}_{1}, \bar{l}_{2}, \bar{l}_{3}, \bar{l}_{4}, \bar{l}_{5}, \bar{l}_{6}, \bar{l}_{7}\right)$ for $\left(\widetilde{u}, \widetilde{p}, \widetilde{q}, \widetilde{\alpha}, \widetilde{s}_{0}, \widetilde{s}_{2}\right) \in \widehat{\mathbf{B}}_{1}$ and $\left(\bar{l}_{1}, \bar{l}_{2}, \bar{l}_{3}, \bar{l}_{4}, \bar{l}_{5}, \bar{l}_{6}, \bar{l}_{7}\right) \in \widehat{\mathbf{B}}_{2}$ if and only if

$$
\left\{\begin{array}{l}
\nu a(\widetilde{u}, \widehat{v})+c(\widetilde{u} ; \widehat{u}, \widehat{v})+c(\widehat{u} ; \widetilde{u}, \widehat{v})+b(\widehat{v}, \widetilde{p})=\int_{\widehat{\Omega}} \bar{l}_{1} \cdot \widehat{v} \mathrm{~d} \vec{x} \quad \forall \widehat{v} \in \mathbf{H}_{0}^{1}(\widehat{\Omega}) \\
b(\widetilde{u}, \widehat{z})=\int_{\widehat{\Omega}} \bar{l}_{2} \widehat{z} \mathrm{~d} \vec{x} \quad \forall \widehat{z} \in L_{0}^{2}(\widehat{\Omega}) \\
\int_{\Gamma_{\alpha}} \widetilde{u} \cdot \vec{s} \mathrm{~d} s+\int_{\Gamma_{\alpha}}(\vec{V}(\widetilde{\alpha}) \cdot \vec{n})\left(k+\frac{\partial}{\partial n}\right) \vec{u} \cdot \vec{s} \mathrm{~d} s=\int_{I} \bar{l}_{3} \cdot \vec{s} \mathrm{~d} s \quad \forall \vec{s} \in \mathbf{H}^{-1 / 2}(I) \\
\int_{\Gamma(\alpha)-\Gamma_{\alpha}} \widetilde{u} \cdot \vec{s} \mathrm{~d} s=\int_{\Gamma(\alpha)-\Gamma_{\alpha}} \bar{l}_{4} \cdot \vec{s} \mathrm{~d} s \quad \forall \vec{s} \in \mathbf{H}^{-1 / 2}\left(\Gamma(\alpha)-\Gamma_{\alpha}\right) \\
\int_{I} \widetilde{q} v \mathrm{~d} x+\int_{I} \frac{\mathrm{d} \widetilde{\alpha}}{\mathrm{d} x} \frac{\mathrm{d} v}{\mathrm{~d} x} \mathrm{~d} x=\int_{I} \bar{l}_{5} v \mathrm{~d} x \quad \forall v \in H_{0}^{1}(I) \\
2 \widetilde{\alpha}\left(\alpha-\alpha_{0}\right)+2 \widetilde{s}_{0} s_{0}=\bar{l}_{6} \quad \forall x \in I \\
2 \widetilde{q}\left(q-q_{0}\right)+2 \widetilde{s}_{2} s_{2}=\bar{l}_{7} \quad \forall x \in I \\
\widetilde{\alpha}(a)=\widetilde{\alpha}(b)=\widetilde{\alpha}^{\prime}(a)=\widetilde{\alpha}^{\prime}(b)=0
\end{array}\right.
$$

where $\vec{V}(\widetilde{\alpha})=(0, \widetilde{\alpha}), \kappa$ denotes the curvature, and $\vec{n}$ is the normal vector to $\Gamma_{\alpha}$. Moreover, the strict derivative of $Q$ at a point $\left(\widehat{u}, \widehat{p}, q, \alpha, s_{0}, s_{2}\right) \in \widehat{\mathbf{B}}_{1}$ is given by the bounded linear operator $Q^{\prime}\left(\widehat{u}, \widehat{p}, q, \alpha, s_{0}, s_{2}\right): \widehat{\mathbf{B}}_{1} \rightarrow \mathbb{R} \times \widehat{\mathbf{B}}_{2}$, where $Q^{\prime}\left(\widehat{u}, \widehat{p}, q, \alpha, s_{0}, s_{2}\right) \cdot\left(\widetilde{u}, \widetilde{p}, \widetilde{q}, \widetilde{\alpha}, \widetilde{s}_{0}, \widetilde{s}_{2}\right)=\left(\bar{a}, \bar{l}_{1}, \bar{l}_{2}, \bar{l}_{3}, \bar{l}_{4}, \bar{l}_{5}, \bar{l}_{6}, \bar{l}_{7}\right)$, for $\left(\widetilde{u}, \widetilde{p}, \widetilde{q}, \widetilde{\alpha}, \widetilde{s}_{0}, \widetilde{s}_{2}\right) \in \widehat{\mathbf{B}}_{1}$ and $\left(\bar{a}, \bar{l}_{1}, \bar{l}_{2}\right.$, $\left.\bar{l}_{3}, \bar{l}_{4}, \bar{l}_{5}, \bar{l}_{6}, \bar{l}_{7}\right) \in \mathbb{R} \times \widehat{\mathbf{B}}_{2}$ if and only if

$$
\left(\begin{array}{c}
\mathcal{J}^{\prime}(\widehat{u}, q, \alpha) \cdot\left(\widetilde{u}, \widetilde{p}, \widetilde{q}, \widetilde{\alpha}, \widetilde{s}_{0}, \widetilde{s}_{2}\right) \\
M^{\prime}\left(\widehat{u}, \widehat{p}, q, \alpha, s_{0}, s_{2}\right) \cdot\left(\widetilde{u}, \widetilde{p}, \widetilde{q}, \widetilde{\alpha}, \widetilde{s}_{0}, \widetilde{s}_{2}\right)
\end{array}\right)=\left(\begin{array}{c}
\bar{a} \\
\left(\bar{l}_{1}, \bar{l}_{2}, \bar{l}_{3}, \bar{l}_{4}, \bar{l}_{5}, \bar{l}_{6}, \bar{l}_{7}\right)
\end{array}\right)
$$

where

$$
\mathcal{J}^{\prime}(\widehat{u}, q, \alpha) \cdot\left(\widetilde{u}, \widetilde{p}, \widetilde{q}, \widetilde{\alpha}, \widetilde{s}_{0}, \widetilde{s}_{2}\right)=\beta \int_{I} q \widetilde{q} \mathrm{~d} x+\nu\left(\int_{\Gamma_{\alpha}} \frac{\partial \widehat{u}}{\partial n} \frac{\partial \widehat{u}}{\partial n}(\vec{V}(\widetilde{\alpha}) \cdot \vec{n}) \mathrm{d} s+2 \int_{\Omega(\alpha)}(\nabla \widehat{u} \cdot \nabla \widetilde{u}) \mathrm{d} \vec{x}\right)
$$

Proof. The linearity of the operator $M^{\prime}\left(\widehat{u}, \widehat{p}, q, \alpha, s_{0}, s_{2}\right)$ is obvious and its boundedness follows from the continuity of the forms $a(\cdot, \cdot), b(\cdot, \cdot)$, and $c(\cdot, \cdot, \cdot)$ combined with the trace theorem for Sobolev spaces. Likewise, the linearity and boundedness of the operator $Q^{\prime}\left(\widehat{u}, \widehat{p}, q, \alpha, s_{0}, s_{2}\right)$ are obvious. The fact that $M^{\prime}\left(\widehat{u}, \widehat{p}, q, \alpha, s_{0}, s_{2}\right)$ is the strict derivative of the mapping $M\left(\widehat{u}, \widehat{p}, q, \alpha, s_{0}, s_{2}\right)$ also follows from the continuity of the trilinear form 
$c(\cdot, \cdot, \cdot)$. Indeed, we have that for any $\left(\widehat{u}, \widehat{p}, q, \alpha, s_{0}, s_{2}\right) \in \widehat{\mathbf{B}}_{1}$ and for all $\left(\widehat{w}, \widehat{r}, \vec{\theta}, \vec{\eta}, \mu, \tau_{0}, \tau_{2}\right) \in \widehat{\mathbf{B}}_{2}^{*}$,

$$
\begin{aligned}
& \left\langle\left(\widehat{w}, \widehat{r}, \vec{\theta}, \vec{\eta}, \mu, \tau_{0}, \tau_{2}\right), M\left(\widehat{u}_{1}, \widehat{p}_{1}, q_{1}, \alpha_{1}, s_{01}, s_{21}\right)-M\left(\widehat{u}_{2}, \widehat{p}_{2}, q_{2}, \alpha_{2}, s_{02}, s_{22}\right)\right. \\
& \left.-M^{\prime}\left(\widehat{u}, \widehat{p}, q, \alpha, s_{0}, s_{2}\right) \cdot\left(\widehat{u}_{1}-\widehat{u}_{2}, \widehat{p}_{1}-\widehat{p}_{2}, q_{1}-q_{2}, \alpha_{1}-\alpha_{2}, s_{01}-s_{02}, s_{21}-s_{22}\right)\right\rangle \\
& =\nu a\left(\widehat{u}_{1}, \widehat{w}\right)+c\left(\widehat{u}_{1} ; \widehat{u}_{1}, \widehat{w}\right)+b\left(\widehat{w}, \widehat{p}_{1}\right)-(\widehat{f}, \widehat{w})+b\left(\widehat{u}_{1}, \widehat{r}\right) \\
& \quad-\left(\nu a\left(\widehat{u}_{2}, \widehat{w}\right)+c\left(\widehat{u}_{2} ; \widehat{u}_{2}, \widehat{w}\right)+b\left(\widehat{w}, \widehat{p}_{2}\right)-(\widehat{f}, \widehat{w})+b\left(\widehat{u}_{2}, \widehat{r}\right)\right) \\
& \quad-\left(\nu a\left(\widehat{u}_{1}-\widehat{u}_{2}, \widehat{w}\right)+c\left(\widehat{u}_{1}-\widehat{u}_{2} ; \widehat{u}, \widehat{w}\right)+c\left(\widehat{u} ; \widehat{u}_{1}-\widehat{u}_{2}, \widehat{w}\right)+b\left(\widehat{w}, \widehat{p}_{1}-\widehat{p}_{2}\right)+b\left(\widehat{u}_{1}-\widehat{u}_{2}, \widehat{r}\right)\right) \\
& \quad+\int_{\Gamma(\alpha)-\Gamma_{\alpha_{1}}} \vec{\eta} \cdot\left(\widehat{u}_{1}-\vec{g}\right) \mathrm{d} s-\int_{\Gamma(\alpha)-\Gamma_{\alpha_{2}}} \vec{\eta} \cdot\left(\widehat{u}_{2}-\vec{g}\right) \mathrm{d} s \\
& \quad-\int_{\Gamma(\alpha)-\Gamma_{\alpha}} \vec{\eta} \cdot\left(\widehat{u}_{1}-\widehat{u}_{2}\right) \mathrm{d} s+\int_{\Gamma_{\alpha_{1}}} \vec{\theta} \cdot \widehat{u}_{1} \mathrm{~d} s-\int_{\Gamma_{\alpha_{2}}} \vec{\theta} \cdot \widehat{u}_{2} \mathrm{~d} s \\
& \quad-\int_{\Gamma_{\alpha}} \vec{\theta} \cdot\left(\widehat{u}_{1}-\widehat{u}_{2}\right) \mathrm{d} s-\int_{\Gamma_{\alpha}}\left(\vec{V}\left(\alpha_{1}-\alpha_{2}\right) \cdot \vec{n}\right) \vec{\theta} \cdot\left(k+\frac{\partial}{\partial n}\right) \widehat{u} \mathrm{~d} s \\
& \quad+\int_{I}^{\tau_{0}}\left(\left(\alpha_{1}-\alpha_{0}\right)^{2}+s_{01}^{2}-\left(\alpha_{2}-\alpha_{0}\right)^{2}-s_{02}^{2}-2\left(\alpha-\alpha_{0}\right)\left(\alpha_{1}-\alpha_{2}\right)\right. \\
& \left.\quad-2 s_{0}\left(s_{01}-s_{02}\right)\right)+\tau_{2}\left(\left(q_{1}-q_{0}\right)^{2}+s_{21}^{2}-\left(q_{2}-q_{0}\right)^{2}-s_{22}^{2}\right. \\
& \left.\quad-2\left(q-q_{0}\right)\left(q_{1}-q_{2}\right)-2 s_{2}\left(s_{21}-s_{22}\right)\right) \mathrm{d} x,
\end{aligned}
$$

where $\vec{V}(z)=(0, z)$. From Lemma 3.1 we have that for every $\epsilon\left\|\alpha_{1}-\alpha_{2}\right\|_{H^{2}} / 4>0$ there exits a $\delta_{1}>0$ such that

$$
\left|\int_{\Gamma_{\alpha_{i}}} \vec{\theta} \cdot \widehat{u}_{i} \mathrm{~d} s-\int_{\Gamma_{\alpha}} \vec{\theta} \cdot \widehat{u}_{i} \mathrm{~d} s-t \int_{\Gamma_{\alpha}}\left(\vec{V}\left(\alpha_{i}-\alpha\right) \cdot \vec{n}\right)\left(\kappa+\frac{\partial}{\partial n}\right)\left(\vec{\theta} \cdot \widehat{u}_{i}\right) \mathrm{d} s\right| \leq \frac{\epsilon}{4}\left\|\alpha_{1}-\alpha_{2}\right\|_{H^{2}(I)}
$$

for $i=1,2$ for all $t \leq \delta_{1}$. Therefore, we have

$$
\begin{aligned}
& \mid\left\langle\left(\widehat{w}, \widehat{r}, \vec{\theta}, \vec{\eta}, \mu, \tau_{0}, \tau_{2}\right), M\left(\widehat{u}_{1}, \widehat{p}_{1}, q_{1}, \alpha_{1}, s_{01}, s_{21}\right)-M\left(\widehat{u}_{2}, \widehat{p}_{2}, q_{2}, \alpha_{2}, s_{02}, s_{22}\right)\right. \\
& \left.-M^{\prime}\left(\widehat{u}, \widehat{p}, q, \alpha, s_{0}, s_{2}\right) \cdot\left(\widehat{u}_{1}-\widehat{u}_{2}, \widehat{p}_{1}-\widehat{p}_{2}, q_{1}-q_{2}, \alpha_{1}-\alpha_{2}, s_{01}-s_{02}, s_{21}-s_{22}\right)\right\rangle \mid \\
& \leq \frac{\epsilon}{2}\left\|\alpha_{1}-\alpha_{2}\right\|_{H^{2}(I)}+\left|c\left(\widehat{u}_{1}-\widehat{u}_{2}, \widehat{u}-\widehat{u}_{1}, \vec{w}\right)+c\left(\widehat{u}-\widehat{u}_{2}, \widehat{u}_{1}-\widehat{u}_{2}, \vec{w}\right)\right| \\
& \quad+t\left(\int_{\Gamma_{\alpha}}\left(\vec{V}\left(\alpha_{1}-\alpha\right) \cdot \vec{n}\right)\left(\kappa+\frac{\partial}{\partial n}\right)\left(\widehat{u}_{1}-\widehat{u}_{2}\right) \cdot \vec{\theta} \mathrm{d} s\right. \\
& \left.\quad+\int_{\Gamma_{\alpha}}\left(\vec{V}\left(\alpha_{1}-\alpha_{2}\right) \cdot \vec{n}\right)\left(\kappa+\frac{\partial}{\partial n}\right)\left(\widehat{u}_{2}-\widehat{u}\right) \cdot \vec{\theta} \mathrm{d} s\right) \\
& \quad+\int_{I}^{\tau_{0}}\left(\left(\alpha_{1}-\alpha_{2}\right)\left(\alpha_{1}-\alpha+\alpha_{2}-\alpha\right)+\left(s_{01}-s_{02}\right)\left(s_{01}-s_{0}+s_{02}-s_{0}\right)\right) \\
& \quad+\tau_{2}\left(\left(q_{1}-q_{2}\right)\left(q_{1}-q+q_{2}-q\right)+\left(s_{21}-s_{22}\right)\left(s_{21}-s_{2}+s_{22}-s_{2}\right)\right) \mathrm{d} x
\end{aligned}
$$


for all $t \leq \delta_{1}$. Then, by using the continuity of the form $c(\cdot, \cdot, \cdot)$, the Sobolev imbedding theorem, and the trace theorem, we have, for some constants $C_{1}, C_{2}, C_{3}, C_{4}>0$, that

$$
\begin{aligned}
& \| M\left(\widehat{u}_{1}, \widehat{p}_{1}, q_{1}, \alpha_{1}, s_{01}, s_{21}\right)-M\left(\widehat{u}_{2}, \widehat{p}_{2}, q_{2}, \alpha_{2}, s_{02}, s_{22}\right) \\
& \left.-M^{\prime}\left(\widehat{u}, \widehat{p}, q, \alpha, s_{0}, s_{2}\right)\right) \cdot\left(\widehat{u}_{1}-\widehat{u}_{2}, \widehat{p}_{1}-\widehat{p}_{2}, q_{1}-q_{2}, \alpha_{1}-\alpha_{2}, s_{01}-s_{02}, s_{21}-s_{22}\right) \|_{\widehat{\mathbf{B}}_{1}} \\
& \leq \frac{\epsilon}{2}\left\|\alpha_{1}-\alpha_{2}\right\|_{2}+C_{1}\left(\left\|\widehat{u}_{1}-\widehat{u}_{2}\right\|_{1}\left\|\widehat{u}-\widehat{u}_{1}\right\|_{1}+\left\|\widehat{u}_{1}-\widehat{u}_{2}\right\|_{1}\left\|\widehat{u}-\widehat{u}_{2}\right\|\right) \\
& +C_{2}\left(\left\|\alpha_{1}-\alpha\right\|_{2}\left\|\widehat{u}_{1}-\widehat{u}_{2}\right\|+\left\|\alpha_{1}-\alpha_{2}\right\|_{2}\left\|\widehat{u}-\widehat{u}_{2}\right\|\right) \\
& +C_{3}\left(\left\|\alpha_{1}-\alpha_{2}\right\|_{2}\left(\left\|\alpha-\alpha_{1}\right\|_{2}+\left\|\alpha-\alpha_{2}\right\|_{2}\right)+\left\|q_{1}-q_{2}\right\|\left(\left\|q-q_{1}\right\|+\left\|q-q_{2}\right\|\right)\right. \\
& \left.+\left\|s_{01}-s_{02}\right\|\left(\left\|s_{0}-s_{01}\right\|+\left\|s_{0}-s_{02}\right\|\right)+\left\|s_{21}-s_{22}\right\|\left(\left\|s_{2}-s_{21}\right\|+\left\|s_{2}-s_{22}\right\|\right)\right) \\
& \leq \frac{\epsilon}{2}\left\|\alpha_{1}-\alpha_{2}\right\|_{2}+C_{4} \|\left(\widehat{u}_{1}-\widehat{u}_{2}, \widehat{p}_{1}-\widehat{p}_{2}, q_{1}-q_{2}, \alpha_{1}-\alpha_{2}, s_{01}-s_{02}, s_{21}-s_{22} \|_{\widehat{\mathbf{B}}_{1}}\right. \\
& \left(\left\|\left(\widehat{u}-\widehat{u}_{1}, \widehat{p}-\widehat{p}_{1}, q-q_{1}, \alpha-\alpha_{1}, s_{0}-s_{01}, s_{2}-s_{21}\right)\right\|_{\widehat{\mathbf{B}}_{1}}\right. \\
& \left.+\left\|\left(\widehat{u}-\widehat{u}_{2}, \widehat{p}-\widehat{p}_{2}, q-q_{2}, \alpha-\alpha_{2}, s_{2}-s_{02}, s_{2}-s_{22}\right)\right\|_{\widehat{\mathbf{B}}_{1}}\right)
\end{aligned}
$$

for $t \leq \delta_{1}$. Then, for any $\epsilon>0$, by choosing $\delta=\epsilon C_{4} / 2$, we have that, whenever $\|\left(\widehat{u}-\widehat{u}_{1}, \widehat{p}-\widehat{p}_{1}, q-q_{1}, \alpha-\right.$ $\left.\alpha_{1}, s_{0}-s_{01}, s_{2}-s_{21}\right) \|_{\widehat{\mathbf{B}}_{1}}<\delta$ and $\left\|\left(\widehat{u}-\widehat{u}_{2}, \widehat{p}-\widehat{p}_{2}, q-q_{2}, \alpha-\alpha_{2}, s_{0}-s_{02}, s_{2}-s_{22}\right)\right\|_{\widehat{\mathbf{B}}_{1}}<\delta$,

$$
\begin{aligned}
& \| M\left(\widehat{u}_{1}, \widehat{p}_{1}, q_{1}, \alpha_{1}, s_{01}, s_{21}\right)-M\left(\widehat{u}_{2}, \widehat{p}_{2}, q_{2}, \alpha_{2}, s_{02}, s_{22}\right) \\
& -M^{\prime}\left(\widehat{u}, \widehat{p}, q, \alpha, s_{0}, s_{2}\right) \cdot\left(\widehat{u}_{1}-\widehat{u}_{2}, \widehat{p}_{1}-\widehat{p}_{2}, q_{1}-q_{2}, \alpha_{1}-\alpha_{2}, s_{01}-s_{02}, s_{21}-s_{22}\right) \|_{\widehat{\mathbf{B}}_{2}} \\
& \quad \leq \epsilon\left\|\left(\widehat{u}_{1}-\widehat{u}_{2}, \widehat{p}_{1}-\widehat{p}_{2}, q_{1}-q_{2}, \alpha_{1}-\alpha_{2}, s_{01}-s_{02}, s_{21}-s_{22}\right)\right\|_{\widehat{\mathbf{B}}_{1}} .
\end{aligned}
$$

Thus, the mapping $M$ is strictly differentiable on all of $\widehat{\mathbf{B}}_{1}$ and its strict derivative is given by $M^{\prime}$.

Using the strict differentiability of the mapping $M$ and Lemma 3.1, it is then easy to show that the mapping $Q$ is also strictly differentiable and that its strict derivative is given by $Q^{\prime}$.

From the mapping (4.6) we can write the Gateaux derivative for our Navier-Stokes system and recover the results of the previous sections. In fact, the solution $(\vec{u}, p)$ of the Navier-Stokes system with boundary defined by $\Gamma(\alpha)$ can be seen as the restriction to $\Omega(\alpha)$ of the function $(\widehat{u}, \widehat{p})$.

Let $\mathbf{B}_{1}$ and $\mathbf{B}_{2}$ be the space generated by all the restrictions from $\widehat{\Omega}$ to $\Omega(\alpha)$ of the functions in $\widehat{\mathbf{B}}_{1}$ and $\widehat{\mathbf{B}}_{2}$ respectively. With test functions in $\mathbf{H}_{0}^{1}(\Omega(\alpha))$ we write the mapping $M^{\prime}\left(\vec{u}, p, q, \alpha, s_{0}, s_{2}\right)$ as

$$
\left\{\begin{array}{l}
\nu a(\widetilde{u}, \vec{v})+c(\widetilde{u} ; \vec{u}, \vec{v})+c(\vec{u} ; \widetilde{u}, \vec{v})+b(\vec{v}, \widetilde{p})=\int_{\Omega(\alpha)} \bar{l}_{1} \cdot \vec{v} \mathrm{~d} \vec{x} \quad \forall \vec{v} \in H_{0}^{1}(\Omega(\alpha)) \\
b(\widetilde{u}, z)=\int_{\Omega(\alpha)} \bar{l}_{2} z \mathrm{~d} \vec{x} \quad \forall z \in L_{0}^{2}(\Omega(\alpha)) \\
\int_{\Gamma_{\alpha}} \widetilde{u} \cdot \vec{s} \mathrm{~d} s+\int_{\Gamma_{\alpha}}(\vec{V} \cdot \vec{n})\left(k+\frac{\partial}{\partial n}\right) \vec{u} \cdot \vec{s} \mathrm{~d} s=\int_{I} \bar{l}_{3} \cdot \vec{s} \mathrm{~d} s \quad \forall \vec{s} \in \mathbf{H}^{-1 / 2}(I) \\
\int_{\Gamma(\alpha)-\Gamma_{\alpha}} \widetilde{u} \cdot \vec{s} \mathrm{~d} s=\int_{\Gamma(\alpha)-\Gamma_{\alpha}} \bar{l}_{4} \cdot \vec{s} \mathrm{~d} s \quad \forall \vec{s} \in \mathbf{H}^{-1 / 2}\left(\Gamma(\alpha)-\Gamma_{\alpha}\right) \\
\int_{I}^{v} v \widetilde{q} \mathrm{~d} x+\int_{I} \frac{\mathrm{d} \widetilde{\alpha}}{\mathrm{d} x} \frac{\mathrm{d} v}{\mathrm{~d} x} \mathrm{~d} x=\int_{I} \bar{l}_{5} v \mathrm{~d} x \quad \forall v \in H_{0}^{1}(I) \\
2 \widetilde{\alpha}\left(\alpha-\alpha_{0}\right)+2 \widetilde{s}_{0} s_{0}=\bar{l}_{6} \quad \forall x \in I \\
2 \widetilde{q}\left(q-q_{0}\right)+2 \widetilde{s}_{2} s_{2}=\bar{l}_{7} \quad \forall x \in I
\end{array}\right.
$$

with $\widetilde{\alpha}(a)=0, \widetilde{\alpha}(b)=0$ and $\widetilde{\alpha}^{\prime}(a)=\widetilde{\alpha}^{\prime}(b)=0$. 
From (4.8), we note that the regularity of the Gateaux derivative cannot be the same as the solution of the Navier-Stokes system. In fact, the boundary conditions for the Gateaux derivative implies a different degree of regularity.

Also, we note that the map $M^{\prime}\left(\vec{u}, p, q, \alpha, s_{0}, s_{2}\right)$ in (4.8) assumes exactly the same values of the restriction of $M^{\prime}\left(\widehat{u}, \widehat{p}, q, \alpha, s_{0}, s_{2}\right)$ to $\Omega(\alpha)$ since no further information is necessary to identify the function over the domain $\Omega(\alpha)$. The use of (4.8) or (4.6) over the domain $\Omega(\alpha)$ is equivalent and therefore in the rest of the paper we always work with these operators on the domain $\Omega(\alpha)$ and use the notation $M^{\prime}\left(\vec{u}, p, q, \alpha, s_{0}, s_{2}\right)$ and $Q^{\prime}\left(\vec{u}, p, q, \alpha, s_{0}, s_{2}\right)$.

Next, we prove some further properties of the derivatives of the mappings $M$ and $Q$.

Lemma 4.2. Let $\left(\vec{u}, p, q, \alpha, s_{0}, s_{2}\right) \in \mathbf{B}_{1}$ denote a solution of the optimal control problem. Then we have

(i) the operator $M^{\prime}\left(\vec{u}, p, q, \alpha, s_{0}, s_{2}\right)$ has closed range in $\mathbf{B}_{2}$;

(ii) the operator $Q^{\prime}\left(\vec{u}, p, q, \alpha, s_{0}, s_{2}\right)$ has closed range in $\mathbb{R} \times \mathbf{B}_{2}$;

(iii) the operator $Q^{\prime}\left(\vec{u}, p, q, \alpha, s_{0}, s_{2}\right)$ is not onto $\mathbb{R} \times \mathbf{B}_{2}$.

Proof. In order to show (i), we shall show that the range of the last three equations in $(4.8)$ is $\left(L^{2}(I) \times H_{0}^{2}(I) \cap\right.$ $\left.H_{0}^{1}(I) \times L^{2}(I)\right)$ and the range of the other equations is closed. At the moment we focus our attention on the last three equations. If $\left(\vec{u}, p, q, \alpha, s_{0}, s_{2}\right)$ is an optimal solution and $\left(\widetilde{u}, \widetilde{p}, \widetilde{q}, \widetilde{\alpha}, \widetilde{s}_{0}, \widetilde{s}_{2}\right) \in \mathbf{B}_{1}$ such that $\widetilde{\alpha}(a)=\widetilde{\alpha}(b)=$ $\widetilde{\alpha}^{\prime}(a)=\widetilde{\alpha}^{\prime}(b)=0$, then the range of the last three equations is clearly a subset of $\left(L^{2}(I) \times H_{0}^{2}(I) \cap H_{0}^{1}(I) \times L^{2}(I)\right)$. Also, for all $\left(\bar{l}_{5}, \bar{l}_{6}, \bar{l}_{7}\right) \in\left(L^{2}(I) \times H_{0}^{2}(I) \cap H_{0}^{1}(I) \times L^{2}(I)\right)$ we can find an element $\left(\widetilde{q}, \widetilde{\alpha}, \widetilde{s}_{0}, \widetilde{s}_{2}\right)$. In fact, since $\left(\vec{u}, p, q, \alpha, s_{0}, s_{2}\right)$ is an optimal solution, then the equation $\left(\alpha-\alpha_{0}\right)^{2}=\alpha_{m}^{2}-s_{0}^{2}$ implies that the quantities $\left(\alpha-\alpha_{0}\right)$ and $s_{0}$ cannot be zero at the same time so that we can always solve the sixth equation with $\widetilde{s}_{0}=0$ and $\widetilde{\alpha}=\bar{l}_{6} / 2\left(\alpha-\alpha_{0}\right)$ or with $\widetilde{\alpha}=0$ and $\widetilde{s}_{0}=\bar{l}_{6} / 2 s_{0}$ for all $\bar{l}_{6} \in H_{0}^{2}(I) \cap H_{0}^{1}(I)$. In an analogous manner, this can also be done for the last equation in (4.8). Also, we note that if $s_{0}=0$, the solution is $\alpha=c_{0}$ or $\alpha=d_{0}$ and if $s_{2}=0$ the solution is $q=c_{2}$ or $q=d_{2}$. The definition of $\mathcal{B}_{a d}$ and the corresponding choice of the constants $c_{0}, d_{0}, c_{2}$, and $d_{2}$ imply the existence of solutions also when $s_{2}$ and $s_{0}$ are both zero.

Thus, the question of the closedness of the range of the operator $M^{\prime}: \mathbf{B}_{1} \rightarrow \mathbf{B}_{2}$ reduces to the like question for the inhomogeneous Stokes operator $\widetilde{S}: \mathbf{H}^{2}(\Omega(\alpha)) \cap \mathbf{H}_{0}^{1}(\Omega(\alpha)) \times H^{1}(\Omega(\alpha)) \cap L_{0}^{2}(\Omega(\alpha)) \rightarrow \mathbf{H}^{-1}(\Omega(\alpha)) \times$ $L_{0}^{2}(\Omega(\alpha)) \times \mathbf{H}^{1 / 2}(\Gamma(\alpha))$ defined as follows: $\widetilde{S} \cdot(\widetilde{w}, \widetilde{p})=\left(\widetilde{l}_{1}, \widetilde{l}_{2}, \widetilde{l}_{2}\right)$ if and only if

$$
\left\{\begin{array}{l}
\nu a(\widetilde{w}, \vec{v})+b(\vec{v}, \widetilde{p})-(\vec{v}, \widetilde{f})=\left\langle\widetilde{l}_{1}, \vec{v}\right\rangle \quad \forall \vec{v} \in \mathbf{H}_{0}^{1}(\Omega(\alpha)) \\
b(\widetilde{w}, z)=\left(\widetilde{l}_{2}, z\right) \quad \forall z \in L^{2}(\Omega(\alpha)) \\
\int_{\Gamma(\alpha)}(\widetilde{w}-\vec{g}) \cdot \vec{s} \mathrm{~d} s=\int_{\Gamma(\alpha)} \widetilde{l}_{3} \cdot \vec{s} \mathrm{~d} s \quad \vec{s} \in \mathbf{H}^{-1 / 2}(\Gamma(\alpha))
\end{array}\right.
$$

where $\vec{f} \in \mathbf{L}^{2}(\Omega(\alpha)), \vec{g} \in \mathbf{H}^{3 / 2}(\Gamma(\alpha))$, and $\Gamma(\alpha)$ is piecewise $C^{1,1}$ with convex corners. The fact that the operator $\widetilde{S}$ has closed range in $\mathbf{H}^{-1}(\Omega(\alpha)) \times L_{0}^{2}(\Omega(\alpha)) \times \mathbf{H}^{1 / 2}(\Gamma(\alpha))$ follows easily from well-known results for the Stokes equations; see, e.g., [37]. We can then conclude that the operator $\widetilde{S}$ has closed range in $\mathbf{B}_{2}$, and, since the operator $M^{\prime}\left(\vec{u}, p, q, \alpha, s_{0}, s_{2}\right)$ is a compact perturbation of the operator $\widetilde{S}$, we have, from the Fredholm theory, that $M^{\prime}\left(\vec{u}, p, q, \alpha, s_{0}, s_{2}\right)$ itself has closed range in $\mathbf{B}_{2}$. Starting from (i), the proof of (ii) and (iii) can be found easily by using the standard techniques in $[18,19,21,23]$.

The first-order necessary condition follows easily from the fact that the operator $Q^{\prime}\left(\vec{u}, p, q, \alpha, s_{0}, s_{2}\right)$ is not onto $\mathbb{R} \times \mathbf{B}_{2}$; see, e.g., [18,22,23].

Theorem 4.3. Given $(\vec{u}, p, q, \alpha) \in \mathcal{A}_{a d}$. If $\left(\vec{u}, p, q, \alpha, s_{0}, s_{2}\right) \in \mathbf{B}_{1}$ is a solution of the optimal shape control problem, then there exists a nonzero Lagrange multiplier $\left(\lambda, \vec{w}, r, \vec{\theta}, \vec{\eta}, \mu, \tau_{0}, \tau_{2}\right) \in \mathbb{R} \times \mathbf{B}_{2}^{*}$ satisfying the Euler equations

$$
\begin{gathered}
\lambda \mathcal{J}^{\prime}(\vec{u}, q, \alpha) \cdot\left(\widetilde{u}, \widetilde{r}, \widetilde{q}, \widetilde{\alpha}, \widetilde{s}_{0}, \widetilde{s}_{2}\right)+\left\langle\left(\vec{w}, r, \vec{\theta}, \vec{\eta}, \mu, \tau_{0}, \tau_{2}\right), M^{\prime}\left(\vec{u}, p, q, \alpha, s_{0}, s_{2}\right) \cdot\left(\widetilde{u}, \widetilde{r}, \widetilde{q}, \widetilde{\alpha}, \widetilde{s}_{0}, \widetilde{s}_{2}\right)\right\rangle=0 \\
\forall\left(\widetilde{u}, \widetilde{r}, \widetilde{q}, \widetilde{\alpha}, \widetilde{s}_{0}, \widetilde{s}_{2}\right) \in \mathbf{B}_{1},
\end{gathered}
$$


where $\langle\cdot, \cdot\rangle$ denotes the duality pairing between $\mathbf{B}_{2}$ and $\mathbf{B}_{2}^{*}$.

\subsection{The optimality system}

Next, we examine the first-order necessary condition (4.10) to derive an optimality system from which optimal states and controls may be determined.

Theorem 4.4. Let $\left(\vec{u}, p, q, \alpha, s_{0}, s_{2}\right) \in \mathbf{B}_{1}$ denote a solution of the optimal control problem. Then, if $s_{0} \neq 0$ and $s_{2} \neq 0, \mu$ is the solution of

$$
\begin{aligned}
\int_{I} \frac{\mathrm{d} \mu}{\mathrm{d} x} \frac{\mathrm{d} \zeta}{\mathrm{d} x} \mathrm{~d} x+\nu \int_{\Gamma_{\alpha}}\left(\frac{\partial \vec{u}}{\partial n} \frac{\partial \vec{u}}{\partial n}-\frac{\partial \vec{u}}{\partial n} \frac{\partial \vec{w}}{\partial n}\right)(\vec{V}(\zeta) \cdot \vec{n}) \mathrm{d} s=0 & \forall \zeta \in H_{0}^{1}(I) \\
\int_{I}(\mu+\beta q) v \mathrm{~d} x=0 & \forall v \in L^{2}(I)
\end{aligned}
$$

for all $\alpha \in H^{2}(I)$ with $\alpha(a)=z_{1}, \alpha(b)=z_{2}$, and $\alpha^{\prime}(a)=\alpha^{\prime}(b)=0$, where $(\vec{w}, r) \in \mathbf{H}_{0}^{1}(\Omega(\alpha)) \times L_{0}^{2}(\Omega(\alpha))$ satisfies the adjoint problem

$$
\left\{\begin{array}{l}
\nu a(\vec{w}, \vec{v})+c(\vec{v} ; \vec{u}, \vec{w})+c(\vec{u} ; \vec{v}, \vec{w})+b(\vec{v}, r)=-2 \nu \int_{\Omega(\alpha)} \nabla \vec{u} \cdot \nabla \vec{v} \mathrm{~d} \vec{x} \quad \forall \vec{v} \in \mathbf{H}_{0}^{1}(\Omega(\alpha)) \\
b(\vec{w}, q)=0 \quad \forall q \in L_{0}^{2}(\Omega(\alpha)) \\
\vec{w} \in \mathbf{H}_{0}^{1}(\Omega(\alpha)) .
\end{array}\right.
$$

If $s_{0}=0$ we have $\alpha=c_{0}$ or $\alpha=d_{0}$. If $s_{2}=0$ we have $q=c_{2}$ or $q=d_{2}$ which gives $\alpha$ through (2.1) and the appropriate boundary conditions.

Proof. We write (4.13) in the following way

$$
\left\{\begin{array}{l}
\nu a(\vec{w}, \vec{v})+c(\vec{v} ; \vec{u}, \vec{w})+c(\vec{u} ; \vec{v}, \vec{w})+b(\vec{v}, r) \\
=\int_{\Gamma_{\alpha}}\left(-r \vec{n}+\nu \frac{\partial \vec{w}}{\partial n}\right) \cdot \vec{v} \mathrm{~d} \vec{x}-2 \nu \int_{\Omega(\alpha)} \nabla \vec{u} \cdot \nabla \vec{v} \mathrm{~d} \vec{x} \quad \forall \vec{v} \in \mathbf{H}^{1}(\Omega(\alpha)) \\
b(\vec{w}, q)=0 \quad \forall q \in L_{0}^{2}(\Omega(\alpha)) .
\end{array}\right.
$$

The first-order necessary condition (4.10) is equivalent to

$$
\begin{aligned}
& \lambda\left(2 \nu \int_{\Omega(\alpha)}(\nabla \vec{u} \cdot \nabla \widetilde{u}) \mathrm{d} \vec{x}+\nu \int_{\Gamma_{\alpha}} \frac{\partial \vec{u}}{\partial n} \frac{\partial \vec{u}}{\partial n}(\vec{V}(\widetilde{\alpha}) \cdot \vec{n}) \mathrm{d} s+\beta \int_{I} \widetilde{q} q \mathrm{~d} x\right) \\
& +\nu a(\widetilde{u}, \vec{w})+c(\widetilde{u}, \vec{u}, \vec{w})+c(\vec{u}, \widetilde{u}, \vec{w})+b(\vec{w}, \widetilde{p})+b(\widetilde{u}, r)+\int_{\Gamma_{\alpha}} \vec{\theta} \cdot\left(\widetilde{u}+(\vec{V}(\widetilde{\alpha}) \cdot \vec{n}) \frac{\partial \vec{u}}{\partial n}\right) \mathrm{d} s \\
& +\int_{\Gamma(\alpha)-\Gamma_{\alpha}} \vec{\eta} \cdot \widetilde{u} \mathrm{~d} s+\int_{I} \mu \widetilde{q} \mathrm{~d} x+\int_{I} \frac{\mathrm{d} \widetilde{\alpha}}{\mathrm{d} x} \frac{\mathrm{d} \mu}{\mathrm{d} x} \mathrm{~d} x+2 \int_{I} \tau_{0}\left(\widetilde{\alpha}\left(\alpha-\alpha_{0}\right)+s_{0} \widetilde{s}_{0}\right)+\tau_{2}\left(\widetilde{q}\left(q-q_{0}\right)+s_{2} \widetilde{s}_{2}\right) \mathrm{d} x=0
\end{aligned}
$$

for all $\left(\widetilde{u}, \widetilde{p}, \widetilde{q}, \widetilde{\alpha}, \widetilde{s}_{0}, \widetilde{s}_{2}\right) \in \mathbf{B}_{1}$. We are free to choose $\lambda=1$.

Choosing $\widetilde{p}=\widetilde{\alpha}=\widetilde{q}=\widetilde{s}_{0}=\widetilde{s}_{2}=0$ in the first-order necessary condition yields (4.14), $\vec{\theta}=r \vec{n}-\nu \frac{\partial \vec{w}}{\partial n}$ on $\Gamma_{\alpha}$, and $\vec{\eta}=\overrightarrow{0}$ on $\Gamma(\alpha)-\Gamma_{\alpha}$. Choosing $\widetilde{u}=\overrightarrow{0}, \widetilde{p}=\widetilde{s}_{0}=\widetilde{\alpha}=\widetilde{s}_{2}=0$ we recover (4.12) and, for $\widetilde{u}=\overrightarrow{0}$, $\widetilde{p}=\widetilde{s}_{0}=\widetilde{q}=\widetilde{s}_{2}=0$, we recover (4.11). Thus, we have shown that this theorem holds for $s_{i} \neq 0$ for $i=0,2$.

If $s_{i}=0$ for some $i$ we have that $\alpha^{(i)}=c_{i}$ or $\alpha^{(i)}=d_{i}$. For example, if $s_{0}=0$ we have $\alpha=c_{0}$ or $\alpha=d_{0}$. If $s_{2}=0$ over an interval $I$ then $\alpha$ should be parabolic with boundary condition determined by $\alpha$ outside $I$. Starting from the value of $\alpha^{(i)}$, all the quantities and in particular $\tau_{i}$ can be easily determined. 
It is obvious that the first-order necessary condition obtained in the previous section, when applicable, is the same as that obtained by the Lagrangian formulation. The introduction of the variable $s_{0}$ and $s_{2}$ gives an insight to the structure of the optimal solution but it is clear that it is not convenient in the numerical computations. Numerically it is difficult to evaluate the variables $s_{0}$ and $s_{2}$ and many local extremal points can be introduced in such an attempt.

As a consequence of the optimality system we have to solve

$$
\begin{gathered}
\left\{\begin{array}{l}
\nu a(\vec{u}, \vec{v})+c(\vec{u} ; \vec{u}, \vec{v})+b(\vec{v}, p)=\langle\vec{f}, \vec{v}\rangle \quad \forall \vec{v} \in \mathbf{H}_{0}^{1}(\Omega(\alpha)) \\
b(\vec{u}, r)=0 \quad \forall r \in L_{0}^{2}(\Omega(\alpha)) \\
\langle\vec{u}, \vec{s}\rangle_{\Gamma(\alpha)}=\langle\vec{g}, \vec{s}\rangle_{\Gamma(\alpha)} \quad \forall \vec{s} \in \mathbf{H}^{1 / 2}(\Gamma(\alpha))
\end{array}\right. \\
\left\{\begin{array}{l}
\nu a(\vec{w}, \vec{v})+c(\vec{v} ; \vec{u}, \vec{w})+c(\vec{u} ; \vec{v}, \vec{w})+b(\vec{v}, r)=-2 \nu \int_{\Omega(\alpha)} \nabla \vec{u} \cdot \nabla \vec{v} \mathrm{~d} \vec{x} \quad \forall \vec{v} \in \mathbf{H}_{0}^{1}(\Omega(\alpha)) \\
b(\vec{w}, r)=0 \quad \forall r \in L_{0}^{2}(\Omega(\alpha)) \\
\vec{w}=\overrightarrow{0} \quad \text { on } \quad \Gamma(\alpha)
\end{array}\right. \\
\int_{I} \frac{\mathrm{d} \mu}{\mathrm{d} x} \frac{\mathrm{d} \zeta}{\mathrm{d} x} \mathrm{~d} x+\nu \int_{\Gamma_{\alpha}}\left(\frac{\partial \vec{u}}{\partial n} \frac{\partial \vec{u}}{\partial n}-\frac{\partial \vec{u}}{\partial n} \frac{\partial \vec{w}}{\partial n}\right)(\vec{V}(\zeta) \cdot \vec{n}) \mathrm{d} s=0 \quad \forall \zeta \in H_{0}^{1}(I) \\
-\int_{I} \frac{\mathrm{d} \alpha}{\mathrm{d} x} \frac{\mathrm{d} v}{\mathrm{~d} x} \mathrm{~d} x=\int_{I} q v \mathrm{~d} x \quad \forall v \in H_{0}^{1}(I)
\end{gathered}
$$

with $\alpha(a)=z_{0}$ and $\alpha(a)=z_{1}$, and

$$
\int_{I}(\beta q+\mu)(\widetilde{q}-q) \geq 0
$$

for all $\widetilde{\alpha} \in \mathcal{Q}_{a d}$ and for all $\widetilde{q} \in \mathcal{B}_{a d}$. The numerical solution of this system of variational equations and inequalities is a rather important question and thus we propose a simple projected gradient algorithm (see [11,24]). In practice, one cannot solve the system simultaneously and at each iteration the method requires the sequential solution of the Navier-Stokes system (4.15) and the adjoint system in (4.16). The solution of the two Poisson equations (4.18) and (4.19) is not very expensive but care should be taken to check that the shape $\alpha$ is in $\mathcal{C}_{a d}$.

\section{REFERENCES}

[1] F. Abergel and R. Temam, On some control problems in fluid mechanics. Theor. Comp. Fluid Dyn. 1 (1990) 303-326.

[2] R. Adams, Sobolev Spaces. Academic Press, New York (1975).

[3] V. Alekseev, V. Tikhomirov and S. Fomin, Optimal Control. Consultants Bureau, New York (1987).

[4] G. Armugan and O. Pironneau, On the problem of riblets as a drag reduction device. Optimal Control Appl. Methods 10 (1989) 93-112.

[5] I. Babuska, The finite element method with Lagrangian multipliers. Numer. Math. 16 (1973) 179-192.

[6] D. Bedivan, Existence of a solution for complete least squares optimal shape problems. Numer. Funct. Anal. Optim. 18 (1997) 495-505.

[7] D. Bedivan and G. Fix, An extension theorem for the space $H_{\text {div }}$. Appl. Math. Lett. (to appear).

[8] D. Begis and R. Glowinski, Application de la méthode des éléments finis à l'approximation d'un problème de domaine optimal. Méthodes de résolution des problèmes approchés. Appl. Math. Optim. 2 (1975) 130-169.

[9] D. Chenais, On the existence of a solution in a domain identification problem. J. Math. Anal. Appl. 52 (1975) $189-219$.

[10] P. Ciarlet, The Finite Element Method for Elliptic Problems. North-Holland, Amsterdam (1978).

[11] P. Ciarlet, Introduction to Numerical Linear Algebra and Optimization. Cambridge University, Cambridge (1989). 
[12] E. Dean, Q. Dinh, R. Glowinski, J. He, T. Pan and J. Periaux, Least squares domain embedding methods for Neumann problems: applications to fluid dynamics, in Domain Decomposition Methods for Partial Differential Equations, D. Keyes et al. Eds., SIAM, Philadelphia (1992).

[13] N. Di Cesare, O. Pironneau and E. Polak, Consistent approximations for an optimal design problem. Report 98005, Labotatoire d'Analyse Numérique, Paris (1998).

[14] N. Fujii, Lower semi-continuity in domain optimization problems. J. Optim. Theory Appl. 57 (1988) 407-422.

[15] V. Girault and P. Raviart, The Finite Element Method for Navier-Stokes Equations: Theory and Algorithms. Springer, New York (1986).

[16] R. Glowinski, Numerical Methods for Nonlinear Variational Problems. Springer, New York (1984).

[17] R. Glowinski and O. Pironneau, Toward the computation of minimum drag profile in viscous laminar flow. Appl. Math. Model. 1 (1976) 58-66.

[18] M. Gunzburger, L. Hou and T. Svobodny, Analysis and finite element approximations of optimal control problems for the stationary Navier-Stokes equations with Dirichlet controls. RAIRO Modél. Math. Anal. Numér. 25 (1991) 711-748.

[19] M. Gunzburger, L. Hou and T. Svobodny, Optimal control and optimization of viscous, incompressible flow, in Incompressible Computational Fluid Dynamics, M. Gunzburger and R. Nicolaides Eds., Cambridge University, New York (1993) 109-150.

[20] M. Gunzburger and H. Kim, Existence of a shape control problem for the stationary Navier-Stokes equations. SIAM J. Control Optim. 36 (1998) 895-909.

[21] M. Gunzburger and S. Manservisi, Analysis and approximation of the velocity tracking problem for Navier-Stokes equations with distributed control. SIAM J. Numer. Anal. 37 (2000) 1481-1512.

[22] M. Gunzburger and S. Manservisi, The velocity tracking problem for Navier-Stokes flows with bounded distributed control. SIAM J. Control Optim. 37 (1999) 1913-1945.

[23] M. Gunzburger and S. Manservisi, A variational inequality formulation of an inverse elasticity problem. Comput. Methods Appl. Mech. Engrg. 189 (2000) 803-823.

[24] M. Gunzburger and S. Manservisi, Some numerical computations of optimal shapes for Navier-Stokes flows (in preparation).

[25] J. Haslinger, K.H. Hoffmann and M. Kocvara, Control fictitious domain method for solving optimal shape design problems. RAIRO Modél. Math. Anal. Numér. 27 (1993) 157-182.

[26] J. Haslinger and P. Neittaanmaki, Finite Element Approximation for Optimal Shape, Material and Topology Design, 2nd edn. Wiley, Chichester (1996).

[27] K. Kunisch and G. Pensil, Shape optimization for mixed boundary value problems based on an embedding domain method (to appear).

[28] O. Pironneau, Optimal Shape Design in Fluid Mechanics. Thesis, University of Paris, France (1976).

[29] O. Pironneau, On optimal design in fluid mechanics. J. Fluid. Mech. 64 (1974) 97-110.

[30] O. Pironneau, Optimal Shape Design for Elliptic Systems. Springer, Berlin (1984).

[31] R. Showalter, Hilbert Space Methods for Partial Differential Equations. Electron. J. Differential Equations (1994) http://ejde.math.swt.edu/mono-toc.html

[32] J. Simon, Domain variation for Stokes flow, in Lecture Notes in Control and Inform. Sci. 159, X. Li and J. Yang Eds., Springer, Berlin (1990) 28-42.

[33] J. Simon, Domain variation for drag Stokes flows, in Lecture notes in Control and Inform. Sci. 114, A. Bermudez Ed., Springer, Berlin (1987) 277-283.

[34] T. Slawig, Domain Optimization for the Stationary Stokes and Navier-Stokes Equations by Embedding Domain Technique. Thesis, TU Berlin, Berlin (1998).

[35] J. Sokolowski and J. Zolesio, Introduction to Shape Optimization: Shape Sensitivity Analysis. Springer, Berlin (1992).

[36] S. Stojanovic, Non-smooth analysis and shape optimization in flow problems. IMA Preprint Series 1046, IMA, Minneapolis (1992).

[37] R. Temam, Navier-Stokes equation. North-Holland, Amsterdam (1979).

[38] R. Temam, Navier-Stokes equations and Nonlinear Functional Analysis. SIAM, Philadelphia (1993).

[39] V. Tikhomirov, Fundamental Principles of the Theory of Extremal Problems. Wiley, Chichester (1986).

To access this journal online:

www.edpsciences.org 